\title{
Revisión y bionomía del género Syncirsodes Butler 1882 (Lepidoptera: Geometridae)
}

\author{
Revision and bionomy of the genus Syncirsodes Butler 1882 (Lepidoptera: Geometridae)
}

PATRICIA A. BOCAZ ${ }^{1} \&$ LUIS E. PARRA ${ }^{2, *}$

\begin{abstract}
${ }^{1}$ Programa Magíster en Ciencias mención Zoología, Departamento de Zoología, Facultad de Ciencias Naturales y Oceanografía, Universidad de Concepción, Casilla 160-C, Concepción, Chile; e-mail: pabocaz@udec.cl

${ }^{2}$ Departamento de Zoología, Facultad de Ciencias Naturales y Oceanográficas, Universidad de Concepción, Casilla 160-C, Concepción, Chile

* Autor para correspondencia: e-mail: luparra@udec.cl
\end{abstract}

\begin{abstract}
RESUMEN
El género Syncirsodes Butler 1882, es redefinido y las especies son taxonómicamente revisadas y redescritas. Las especies que conforman el género son las siguientes: S. primata (Walker 1862), S. subornata (Walker 1863), S. straminea (Butler 1882), S. distictaria (Mabille 1885) y S. hyadesi (Mabille 1885). El ejemplar alotipo de la especie A. primata Walker (1862) corresponde a la hembra de S. distictaria. Las especies deustata Felder \& Rogenhofer (1875) y arenosa, carnea, cinerea, squamosa y turbida descritas por Butler (1882) son los sinónimos junior de S. primata Walker (1862). La ausencia de la armadura genital del material tipo y la falta de otros ejemplares nos hace mantener como válida la especie S. subornata. El género se distribuye principalmente en la región Andina, solo S. subornata se encuentra en la provincia Pampa. Se describen además los estados inmaduros y la bionomía de $S$. distictaria y $S$. primata. Se entregan claves, dibujos de la genitalia, la distribución y fotografías de las diferentes especies.
\end{abstract}

Palabras clave: S. distictaria, S. hyadesi, S. primata, S. straminea, S. subornata, taxonomía, biología, Ennominae, Chile.

\section{ABSTRACT}

The genus Syncirsodes Butler 1882 is redefined and its species are taxonomically revised and described. The species are as follows: S. distictaria (Mabille 1885), S. primata (Walker 1862), S. hyadesi (Mabille 1885), $S$. straminea (Butler 1882) and S. subornata (Walker 1863). The specimen allotype of A. primata Walker (1862) corresponds to the female of $S$. distictaria. The species deustata Felder \& Rogenhofer (1875) and arenosa, carnea, cinerea, squamosa and turbida described by Butler (1882) are junior synonyms of S. primata Walker (1862). The absence of the genitalia of the type and the lack of other specimens make us maintain to $S$. subornata as a valid species. The genus is distributed mainly in the Andean region, only S. subornata is in the Pampa province. Immature stages and bionomy of $S$. distictaria and $S$. primata are described, and an identification key, data on the distribution as well illustrations of the adult moths are provided.

Key words: S. distictaria, S. hyadesi, S. primata, S. straminea, S. subornata, taxonomy, biology, Ennominae, Chile.

\section{INTRODUCCIÓN}

En Chile la familia Geometridae representa el segundo grupo de lepidópteros con mayor diversidad de especies después de la familia Noctuidae (Artigas 1994). De las seis subfamilias que la conforman, cinco están presentes en nuestro país, con 87 géneros, y un total de 320 especies descritas. Ennominae es la subfamilia más diversa a nivel Neotropical (Hepp- ner 1991, Scoble 1995), y también lo es en Chile (Parra 1995, Parra 1999a, Parra \& Vargas 2000). En el último tiempo se han realizado revisiones de algunos géneros de esta subfamilia, entre ellos Psilaspilates (Parra 1999a), Euclidiodes (Parra 1999b) y Neorumia (Parra \& Vargas 2000), contribuyendo al correcto reconocimiento de sus especies y al ordenamiento taxonómico de estos grupos. Esto corresponde a una pequeña fracción del total 
de géneros señalados para Chile, restando aún una serie de revisiones que permitan tener un mejor conocimiento de los distintos taxa que conforman la subfamilia Ennominae. Al mismo tiempo, pocas revisiones reúnen aspectos biológicos de estos organismos.

Dentro de la inmensa diversidad observada en este grupo, el género Syncirsodes se destaca porque sus especies se caracterizan por presentar una gran variabilidad interespecífica e intraespecífica, lo que llevó a Butler (1882 y 1893) a confundir las distintas especies. Este fenómeno se ha mantenido a través del tiempo, en la que diversos autores han seguido las descripciones originales o han utilizado los pocos cambios taxonómicos propuestos recientemente.

Syncirsodes fue creado por Butler (1882) para la especie chilena Syncirsodes straminea, basándose en las formas del cuerpo, antenas y en la coloración de las alas. Felder \& Rogenhofer (1875) describen una nueva especie para Chile Paragonia deustata, muy similar a $S$. straminea. Posteriormente Butler (1882) describe cinco especies de Paragonia incluyendo la especie de Felder \& Rogenhofer (1875). Mabille (1885) describe dos especies más en los géneros Hippoplectis y Ennomos, muy cercanas y similares a Paragonia. Más tarde, Butler (1893) crea el género Anisogonia, en el cual incluye las especies determinadas como Paragonia. Warren (1894), redescribe el género Anisogonia agregando a este las especies Aspilates? primata Walker 1862 y Macaria subornata Walker 1863. Scoble (1999) en su catálogo mundial de la familia Geometridae, señala que el género Ennada es el sinónimo senior del género Anisogonia e incluye dentro de este a las siguientes especies: E. deustata, E. distictaria, E. flavaria, E. hyadesi, E. primata y E. subornata. Finalmente, Pitkin (2002) en su revisión de los géneros neotropicales de Ennominae, indica que Anisogonia es el sinónimo junior del género Syncirsodes y que Ennada es un género monotípico que pertenecería a Larentiinae. De esta manera las especies incluidas en Ennada por Scoble (1999) son incorporadas a Syncirsodes (Pitkin 2002): S. deustata, S. distictaria, $S$. hyadesi, S. primata, S. straminea y S. subornata.

Syncirsodes posee un patrón de coloración de las alas muy variable, característica que dificulta la correcta identificación de sus distintas especies. Sin embargo, el análisis morfológico de la armadura genital de ambos sexos entregan los caracteres diagnósticos para las diferentes especies. El objetivo de este trabajo es entregar una revisión taxonómica que permita un correcto reconocimiento de cada una de las especies, además de dar a conocer aspectos de la bionomía del género Syncirsodes, a través del análisis morfológico de los adultos de las distintas especies, los estados inmaduros e historia natural de $S$. distictaria y $S$. primata como contribución al conocimiento de nuestra biodiversidad y para comprender la estrecha relación de estos insectos con su hábitat.

\section{MATERIALES Y MÉTODOS}

Para la revisión taxonómica en este estudio se examinaron 87 ejemplares adultos provenientes de colectas en terreno, adultos capturados con trampas de luz en la zona de Hualpén (Octava Región de Chile) y ejemplares depositados en el Museo Nacional de Historia Natural, Santiago, Chile (MNHN), Museo de Zoología de la Universidad de Concepción, Concepción, Chile (MZUC), tipos provenientes del Museum Nacional d'Histoire Naturelle, París, Francia (MNHNP) y The Natural History Museum, London, United Kingdom (BMNH). Para cada una de las especies se analizó la armadura genital siguiendo las técnicas descritas por Parra (1991), excepto para la especie S. subornata, puesto que el ejemplar tipo está incompleto y su genitalia perdida. Para la nomenclatura de la genitalia se siguió a Klots (1970) y para la maculación de las alas a Scoble (1995).

Para conocer la biología de las distintas especies se recolectaron larvas en la Península de Hualpén (Octava Región), las que se mantuvieron en una cámara de crianza, en condiciones de temperatura, humedad y fotoperíodo similares a lo observado en el medio natural. Las épocas de vuelo se obtuvieron de la captura de ejemplares en terreno, de los adultos emergidos en laboratorio, y de las etiquetas de los ejemplares estudiados y depositados en los distintos museos. Los huevos se obtuvieron de hembras capturadas en terreno y emergidas en el laboratorio. Los distintos estadios larvales se determinaron por las sucesivas mudas de las larvas, desde la eclosión del huevo hasta la última exuvia larval. En el análisis morfológico de huevos y larvas se utilizó microscopía electrónica de barrido. Para el estudio y nomenclatura de las 
estructuras del huevo se siguió a Salkeld (1983); para las larvas (cápsulas cefálicas, quetotaxia, etc.) a Sterh (1987); y pupas (cremaster, sensilas, etc.) las técnicas descritas por Scoble (1995). Los huevos, larvas y adultos obtenidos fueron depositados en el Museo de Zoología de la Universidad de Concepción, Concepción, Chile (MZUC).

\section{RESULTADOS}

\section{Descripción de los imagos Butler 1882}

Syncirsodes Butler, 1882.

Syncirsodes Butler 1882, p 464; Pitkin 2002 p. 317.

Anisogonia Butler 1893: 459; Warren 1894: 454; Pitkin 2002: 317

Especie tipo. Syncirsodes straminea Butler 1882, p. 464

Diagnosis. La apariencia externa como los caracteres de la genitalia del macho, hacen a Syncirsodes muy similar a los géneros Tetracis Guenée y Microclysia Butler. Sin embargo, un análisis más minucioso nos muestra que en Syncirsodes la genitalia del macho tiene microespinas en la yuxta y un grupo de espinas o cerdas en el área del cucullus; ambos caracteres nos permiten discriminar fácilmente este taxón de los otros dos géneros. La forma de las alas es otro carácter que permite la separación entre estos géneros. En Syncirsodes las alas tienen los bordes externos distintos entre machos y hembras; las hembras tienen una escotadura al nivel de la vena M3, en los machos es liso. En Tetracis las alas tienen el borde externo del primer tercio del ala cóncava en ambos sexos. $M i$ croclysia, en cambio, se distingue porque las alas son más estrechas y ambos sexos tienen antenas simples.

Monofilia. La comparación con los géneros Tetracis y Microclysia (grupos externos) permite establecer de que la presencia de microespinas en la yuxta de la genitalia del macho (Pitkin 2002) y la escotadura en las alas anteriores de la hembra, son las autapomorfías que sustentan la monofilia del género (Bocaz \& Parra, resultados no publicados).

Redescripción del género. Macho: cuerpo gris amarillento pálido, delgado; cabeza y frente del mismo color que las alas anteriores, antenas pectinadas, más claras que la cabeza, palpos de color similar al tórax, segmento medio con escamas largas, 3 veces más largo que el primer segmento. Fórmula tibial 0-2-4; tórax similar en color a las alas anteriores. Abdomen delgado, último segmento abdominal rodeado con un cinturón de escamas largas. La maculación de las alas es muy variable, va desde el amarillo pálido al gris ceniciento. Las alas anteriores están marcadas por dos líneas bien definidas, una cruza el área de la banda postmedial o la banda terminal y postmedial, siempre más oscura que la coloración general, la otra ondulada cerca del área basal; banda medial insconspicua; mancha discal pequeña y oscura. Alas posteriores de color uniforme, similar al de las alas anteriores, línea extradiscal presente.

Hembra: similar al macho en el patrón de color, a diferencia de este las antenas son simples y alas anteriores tienen una gran escotadura en la zona media del borde externo, a nivel de la vena M3.

Genitalia del macho. Uncus subtriangular o espatulado; gnathos generalmente en forma de "U" o "V", ápice con un conjunto de pequeñas espinas dispuestas de manera longitudinal, yuxta parcial o completamente ornamentada con microespinas; socius ausente; valvas subcuadrangulares o rectangulares; saccus subcircular. Aedeagus tubular, subigual al largo de la valva o la mitad de esta; vesica armada con dos conjuntos de espinas cónicas, las posteriores dos veces más largas que las anteriores.

Genitalia de la hembra. Bursa copulatrix elongada; corpus bursae con la región anterior globosa y membranosa, la región posterior eclerosada y estriada; signum ausente; ductus bursae esclerosado, 3 veces más ancho que largo; apófisis posteriores dos veces más largas que las anteriores.

Distribución (Fig. 12). El género se distribuye en la vertiente occidental de los Andes desde Valparaíso por el norte hasta Tierra del Fuego por el sur. En Argentina hay registros en Neuquén y un registro más al norte en Montevideo, Uruguay.

Observaciones: Las especies incluidas en este género tienen un marcado dimorfismo sexual, dado principalmente por la escotadura en las alas anteriores y por las antenas simples que exhiben las hembras (pectinadas en los machos).

El patrón de coloración de las alas de los machos en las distintas especies es muy varia- 
ble, aunque $S$. straminea y $S$. distictaria son las que mantienen un patrón de coloración prácticamente constante. Entre $S$. primata y $S$. hyadesi la variación del patrón antes señalado es mucho mayor, la identificación de las especies sobre la base de la maculación de las alas no es confiable y se debe recurrir al análisis de la genitalia. Este fenómeno se da en menor grado en las hembras, ya que se les puede identificar sin mayores problemas, aunque entre $S$. primata y $S$. hyadesi la coloración anaranjada de las polillas puede provocar confusión, ambas se pueden distinguir porque la banda medial de $S$. primata es siempre más oscura y en $S$. hyadesi es más clara que el patrón de coloración general.

En el Anexo 1 se encuentra una clave construida sobre la base de la maculación y de las armaduras genitales para distinguir las distintas especies de Syncirsodes.

Syncirsodes distictaria (Mabille 1885) (Fig. 1 $A-D)$

Hippoplectis distictaria Mabille 1885, p. 67. Angulo y Casanueva 1981, p. 21.

Ennada distictaria (Mabille) Scoble 1999, p. 269.

Syncirsodes distictaria (Mabille) Pitkin 2002, p. 318. (Pero citada como disticlaria, ortografía incorrecta subsecuente).

Aspilates? primata Walker 1862 (tipo hembra).

Material tipo: Hippoplectis distictaria Mabille 1885. Holotipo examinado (macho) (MNHNP) Insulis Magellanicis (Tierra del Fuego): (Oushuouaya). Aspilates? primata Walker 1862, Alotipo examinado (hembra) (BMNH).

Diagnosis. El macho $S$. distictaria a diferencia del resto de las especies del género, se distingue por su coloración brillante y la presencia de líneas oblicuas grises o castañas oscuras, que cruzan las alas anteriores. La hembra se distingue fácilmente de las otras especies, porque en la zona media del margen externo presenta dos manchas circulares oscuras y además por la coloración anaranjada de la banda medial.

La genitalia del macho se diferencia de las otras especies porque las valvas son anchas y el cucullus presenta escasa cerdas. En la genitalia de la hembra, el ductus bursae está fuertemente esclerosado en los bordes del ostium bursae.
Redescripción. Macho (Fig. 1A): cabeza blanca; frente castaña con escamas amarillentas; antenas pectinadas, ocres, palpos castaños, segmento medio tres veces más largo que el distal; patas ocre con algunas escamas castañas dispersas en la superficie.

Alas anteriores amarillo pálido, brillante; banda terminal con dos manchas circulares oscuras en la zona media del borde externo; una línea castaña o gris oscura cruza la banda adterminal desde el primer cuarto del margen costal y hasta la mitad del margen interno; una segunda línea oblicua nace desde la zona media del margen costal y llega hasta la del margen interno, con una línea ondulada castaña o gris, en el borde interno de la banda medial. Alas posteriores, del mismo color que las anteriores, levemente más pálida; línea extradiscal oscura, se extiende desde la zona media del margen interno del ala hasta la zona media del margen costal.

Hembra (Fig. 1D): Las alas anteriores con una escotadura en la mitad del margen externo, antenas simples. Alas anteriores amarillo pálido; banda terminal y postmedial amarillo pálido, abigarradas con escamas negras en el ápice del ala y sector de la escotadura; banda terminal con dos manchas circulares castaño oscuro; banda medial más oscura, con manchas anaranjadas a castañas en la zona central de esta banda; mancha discal oscura; línea oblicua gris cruza la banda postmedial desde el margen costal hasta la zona media del margen interno, borde externo de la banda antemedial irregular. Alas posteriores amarillo pálido salpicada de escasas escamas castaño claro, línea extradiscal castaña, perceptible desde la zona media del margen interno hasta la zona media del margen costal, mancha discal presente, interceptada por la línea extradiscal. Abdomen levemente más ancho que el macho. Extensión de las alas: 35 a $42 \mathrm{~mm}(\mathrm{n}=23)$.

Genitalia del macho (Fig. 2B y 2C): uncus triangular; gnathos en forma de "U”, ápice agudo con un conjunto de espinas muy pequeñas cónicas y cortas; valvas simples subtriangular, con escasas cerdas, dos veces más largas que anchas; yuxta ornamentada con pequeñas microespinas; saccus subcircular. Aedeagus tubular; vesica armada con dos conjuntos de espinas, la anterior con espinas cortas y cónicas, la posterior con espinas tres veces más largas que las posteriores. 

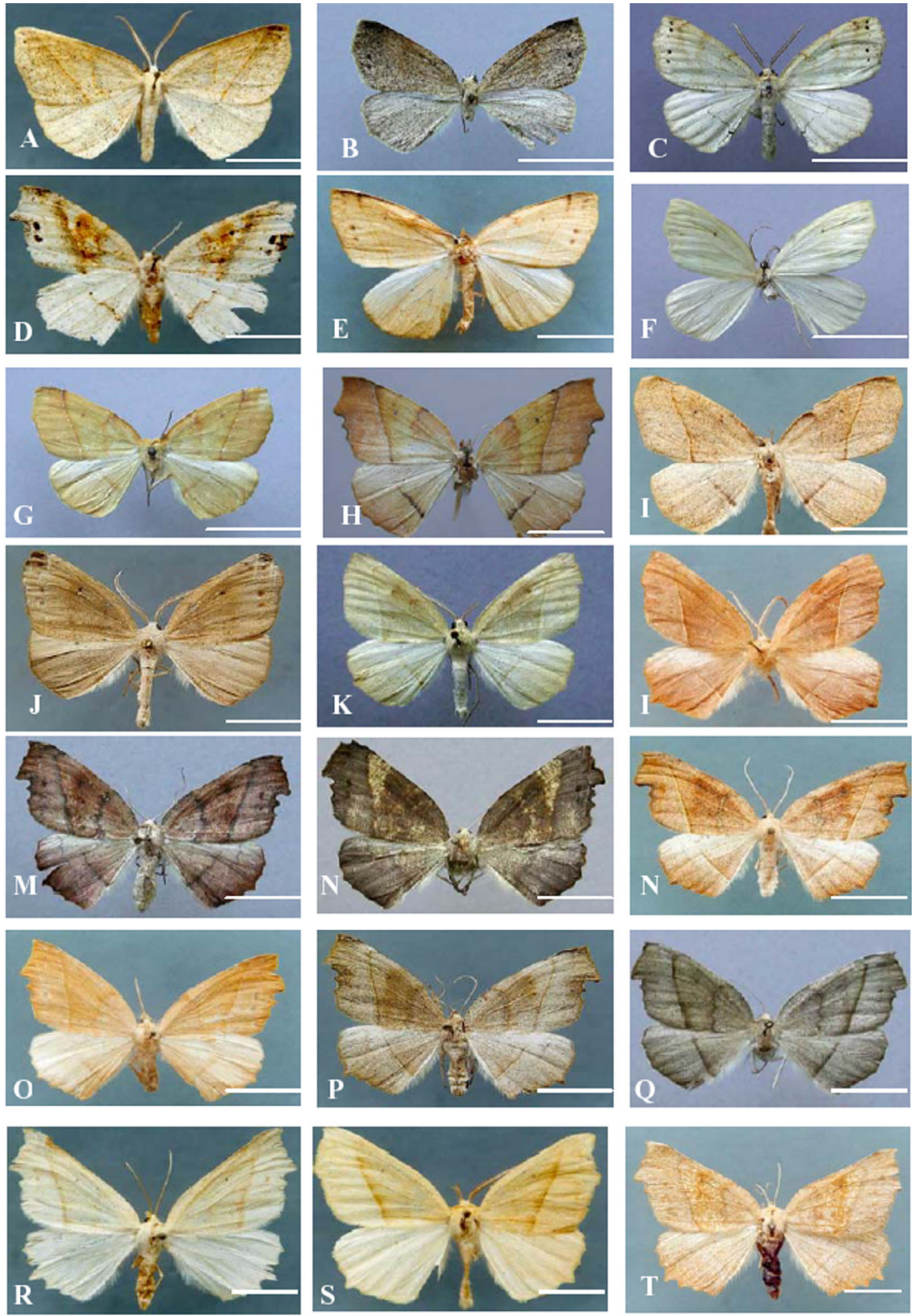

Fig. 1: Adultos de las especies de Syncirsodes. S. distictaria (A, B y C = machos) (D = hembra); $S$. hyadesi $(\mathrm{E}, \mathrm{F}$ y $\mathrm{G}=$ machos $)(\mathrm{H}=$ hembra $) ; S$. primata $(\mathrm{I}, \mathrm{J}, \mathrm{K}$ y L $=$ machos $)(\mathrm{M} \mathrm{N}, \tilde{\mathrm{N}}, \mathrm{O}, \mathrm{P}$ y $\mathrm{Q}=$ hembras); S. straminea $(\mathrm{R}=$ hembra) $(\mathrm{S}$ y $\mathrm{T}=$ machos). Escala: $1 \mathrm{~cm}$.

Adult of Syncirsodes species. S. distictaria (A, B y C = males) $(\mathrm{D}=$ female); S. hyadesi $(\mathrm{E}, \mathrm{F}$ y $\mathrm{G}=\mathrm{male})(\mathrm{H}=\mathrm{female}) ; S$. primata $(\mathrm{I}, \mathrm{J}, \mathrm{K}$ y L = males) $(\mathrm{M} \mathrm{N}, \tilde{\mathrm{N}}, \mathrm{O}, \mathrm{P}$ y Q $=$ females $) ; S$. straminea $(\mathrm{R}=$ female $)(\mathrm{S}$ y $\mathrm{T}=$ males $) . S \mathrm{Scale} 1 \mathrm{~cm}$. 

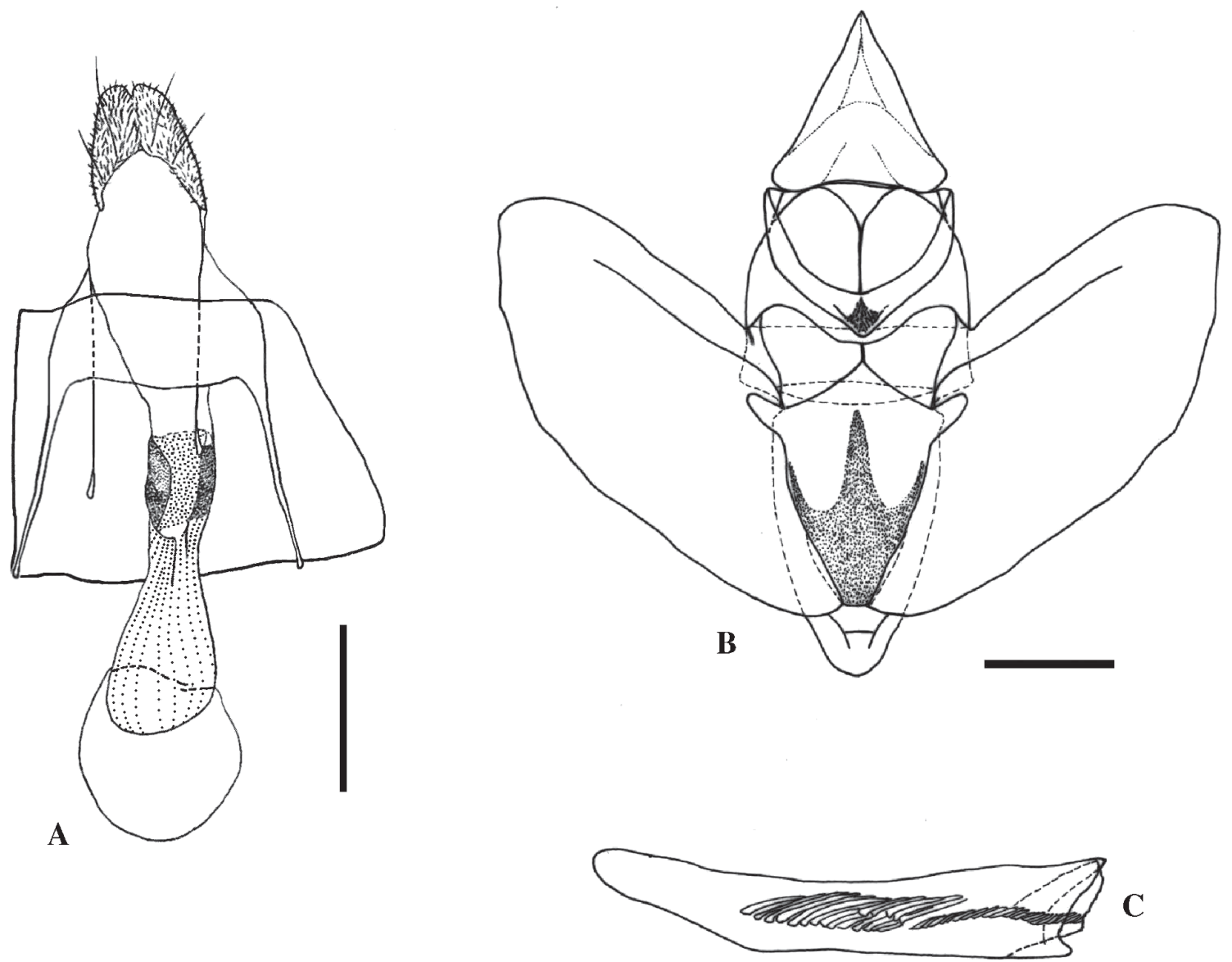

Fig. 2: Syncirsodes distictaria. (A) genitalia hembra en vista ventral, (B) genitalia macho en vista ventral y $(C)$ aedeagus en vista lateral. Escala $1 \mathrm{~mm}$.

Syncirsodes distictaria. (A) female genitalia in ventral view, (B) male genitalia in ventral view and (C) aedeagus in lateral view. Scale $1 \mathrm{~mm}$.

Genitalia de la hembra (Fig. 2A): bursa copulatrix elongada; corpus bursae globoso y membranoso en la región anterior, tubular en la región posterior, con estriaciones longitudinales, ductus bursae angosto y muy esclerosado.

Distribución (Fig.12). Desde la provincia de San Antonio hasta la provincia de Magallanes.

Observaciones. Walker (1862) describe sobre la base de un macho y una hembra la especie Aspilates? primata. La revisión de los tipos, nos permiten concluir que el ejemplar hembra de la serie tipo de esta especie corresponde en realidad a Hippoplectis distictaria Mabille 1885 , por lo que A. primata es un sinónimo de esta última especie. Dentro del género, $S$. distictaria presenta un mayor grado de variación en color entre macho y hembra, ya que la hembra es muy distinta del macho en el patrón de maculación alar. Entre los machos existe variación en la maculación alar, aunque no es tan extrema como otras especies del género. Hay machos con las alas amarillo pálido, salpicado por escamas oscuras en toda la superficie alar y sin la segunda línea oblicua (Fig. 1A); otros tienen alas castaño abigarrado de escamas negras, sin ninguna línea perceptible (Fig. 1B); y por último, existen individuos de color amarillo pálido brillante con las tres líneas oblicuas muy notorias (Fig. 1C). Las manchas circulares del margen terminal pueden o no estar presente.

La genitalia del macho es la más simple respecto a las otras especies del género, ya que no presenta espinas en el sector del cucullus y las valvas son más anchas que en el resto de las especies. La genitalia de la hembra es también muy simple con relación a la genitalia de las otras especies. 
Scoble (1999) y Pitkin (2002) nombran a esta especie como $S$. disticlaria; sin embargo, Mabille (1885) en su publicación original la cita como distictaria, por ello consideramos que Pitkin y Scoble cometen un error ortográfico cuando se refieren a ella.

Material examinado: (14 machos, 11 hembras): Chile: Alotipo, hembra (Aspilates? primata Walker 1862) (BMNH). San Antonio: El Quisco Santiago 2 machos 12-11-50 (MZUC); Algarrobo 1 macho 5-1-1950 (MZUC); Concepción: 1 hembra emergida 11-11-00 P. Bocaz (MZUC); Cautín. Termas de Río Blanco: 4 machos 3-51 (MZUC); Valdivia: Valdivia 1 macho 1891 (4993), 2 hembras 1891 col. Paulsen (4998); 1 hembra (V. Izquierdo) (4999) (MNHN); Chiloé: Quellón 1 hembra enero 1951 J. S. Vargas (MZUC); Última Esperanza: Puerto Natales 2 machos febrero-1953 Alarcón col; 1 macho 18 febrero 1973 M. Cerda col.; 1 hembra febrero-1953 Alarcón col; Magallanes 2 hembras, 17-II-52, T. Cekalovic (MZUC); Isla Guarello 1 hembra 20-marzo-1976 Trampas col. (MZUC); Magallanes: Tres Puentes 1 macho diciembre-1952 Rodríguez col. ; 1 hembra diciembre-1952 Rodríguez col. (MZUC); Holotipo macho Insulis Magellanicis (Tierra del Fuego): (Oushuouaya) (MNHNP).

Syncirsodes hyadesi (Mabille 1885) (Fig. 1E-H)

Ennomos hyadesi Mabille 1885: 65 (pero citada como Eunomos, error ortográfico subsecuente); Angulo \& Casanueva 1981: 12; Pitkin 2002: 318.

Ennada hyadesi (Mabille) Scoble 1999: 269.

Syncirsodes hyadesi (Mabille) Pitkin, 2002: 318. (citada como hyadesii, error ortográfico subsecuente).

Material tipo: Ennomos hyadesi Mabille 1885. Holotipo examinado (hembra) (MNHNP), Insulis magellanicis (Tierra del Fuego).

Diagnosis: los machos y hembras pueden ser confundidos con el fenotipo amarillento o anaranjado y con el castaño salpicado de escamas oscuras de $S$. primata sin embargo, la banda medial es siempre más clara en $S$. hyadesi. En todo caso la armadura genital es la que entrega las principales diferencias. La genitalia del macho se distingue por las valvas rectangulares y un grupo de espinas en su margen costal. La genitalia de la hembra presenta el ductus bursae fuertemente esclerosado, corto y en forma de cinturón.

Redescripción: macho (Fig. 1E): Cabeza amarillenta o anaranjada; frente castaña; antenas pectinadas castaño claro, palpos castaños, segundo segmento tres veces más largo que el proximal. Tórax y patas ocre, con algunas escamas castañas dispersas en la superficie. Abdomen del mismo color que las alas, último segmento abdominal rodeado por pelos largos.

Alas anteriores anaranjadas, dos puntos negros en la zona media del borde externo; banda postmedial interrumpida por una línea oblicua más oscura que la coloración general; banda medial muy tenue; mancha discal oscura y muy pequeña. Alas posteriores, más pálidas que las anteriores, presentan una línea extradiscal más oscura, desde el margen costal al margen interno; mancha discal apenas perceptible en forma de punto.

Hembra (Fig. 1H): similar al macho. Antenas simples; alas anteriores con una escotadura en la zona media del margen externo. Banda medial siempre más clara que la banda postmedial. Extensión de las alas: 35 a 42mm $(\mathrm{n}=8)$.

Genitalia del macho (Fig. 3B-C): uncus espatulado; gnathos en forma de U, ápice terminado en una punta corta y aguda, con un conjunto de microespinas; yuxta ornamentada con microespinas; socius ausente; valvas subtriangulares, con dos grupos de espinas, uno en la zona del cucullus, el otro grupo se ubica en el margen costal de la valva, en número de cinco; saccus subcircular. Aedeagus tubular; vesica armada con dos conjuntos de espinas cónicas, las posteriores tres veces más largas que las anteriores.

Genitalia de la hembra (Fig. 3A): bursa copulatrix elongada, corpus bursae con la región anterior globosa y membranosa, región posterior fuertemente esclerosada y estriada; signum ausente; ductus bursae esclerosado, corto y en forma de cinturón, apófisis posteriores dos veces más largas que las anteriores.

Distribución (Fig. 12): desde la provincia de Cautín hasta la provincia de Magallanes.

Observaciones: la genitalia del macho es la más compleja dentro del grupo de especies y es la única que presenta dos grupos de espinas en las valvas. El patrón de maculación alar varía desde el amarillo al anaranjado. Los machos muestran variación en la maculación alar, hay ejemplares con: (1) las alas anteriores y poste- 

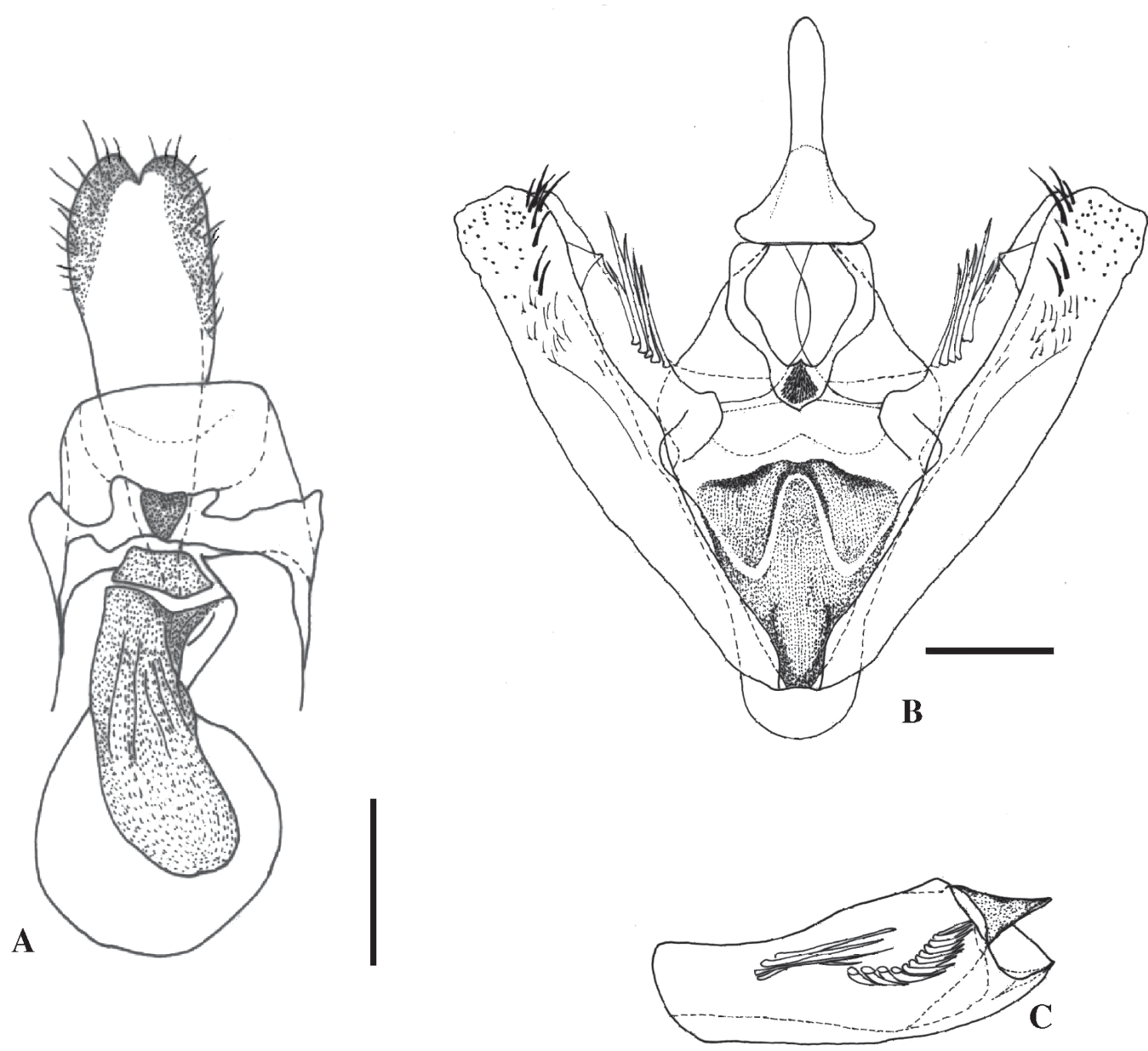

Fig. 3: Syncirsodes hyadesi. (A) genitalia hembra en vista ventral, (B) genitalia macho en vista ventral, y $(\mathrm{C})$ aedeagus en vista lateral. Escala $1 \mathrm{~mm}$.

Syncirsodes hyadesi. (A) female genitalia in ventral view, (B) male genitalia in ventral view, and (C) aedeagus in lateral view. Scale $1 \mathrm{~mm}$.

riores amarillo pálido y con la banda medial apenas perceptible (Fig. 1F); (2) otros con alas anteriores anaranjadas y dos manchas circulares en la zona media del borde externo del ala, presentes o no, línea oblicua transversal, alas posteriores más pálidas (Fig. 1E y 1G); y (3) con alas anteriores y posteriores castañas abigarradas de escamas castaño oscuro y castaño rojizo, línea oblicua transversal castaño oscura. Hembras siempre anaranjadas con la banda medial más clara, banda terminal y postmedial abigarradas de algunas escamas grises, con o sin la presencia de dos manchas circulares en la zona media del borde externo; alas posteriores amarillo pálidas.
En la publicación original, Mabille (1885) dedica la presente especie al Dr. Hyades citándola como E. hyadesi. Posteriormente, Scoble (1999) y Pitkin (2002) citan la especie como $S$. hyadesii, cometiendo un error ortográfico subsecuente.

Material examinado: (5 machos, 4 hembras). Cautín: Termas de Río Blanco 1 hembra 3. 51 (MZUC); Valdivia: Valdivia 1 macho (4788), 1 hembra (4758) (MNHN); Chiloé: Chepu 1 macho L. E. Peña col. 2.52 (MZUC); 1 macho L. E. Peña col. I. 52 (MZUC); Última Esperanza: Isla Guarello 1 hembra 19-mayo-1976 Sanhueza col. (MZUC); Magallanes: Reserva Nacional 
Magallanes. 1 macho, col. J. Petersen (Trampa Malaise) 27-31-XII-1978; Tres Puentes 1 macho Dic-1952 Rodríguez col. (MZUC); Holotipo hembra, Insulis magellanicis (Tierra del Fuego) (MNHNP).

Syncirsodes primata (Walker 1862) (Fig. 1I-Q)

Aspilates? primata Walker 1862, (24): 1076; Scoble 1999: 269; Pitkin 2002: 318; Scoble 1999: 269 (Ennada); Pitkin 2002: 318 (Syncirsodes).

Paragonia deustata Felder \& Rogenhofer 1875: pl.124, fig. 8; Butler 1882: 477; Angulo \& Casanueva 1981: 14; Scoble 1999: 269 (Ennada); Pitkin 2002: 318 (Syncirsodes) sin. nov.

Paragonia arenosa Butler 1882, p. 351; Angulo \& Casanueva 1981: 14. sin. nov.

Paragonia carnea Butler 1882, p. 353; Angulo \& Casanueva 1981: 14. sin. nov.

Paragonia cinerea Butler 1882, p. 354; Angulo \& Casanueva 1981: 14. sin. nov.

Paragonia squamosa Butler 1882, p. 352; Angulo \& Casanueva 1981: 14. sin. nov.

Paragonia turbida Butler1882, p. 352; Angulo \& Casanueva 1981: 14. sin. nov.

Material tipo: Aspilates? primata Walker 1862, holotipo examinado (macho) (BMNH); Chile. P. deustata Felder \& Rogenhofer 1875, holotipo examinado (hembra) (BMNH); P. arenosa Butler 1882, holotipo y alotipo examinados (macho y hembra), Valparaíso, Chile (MBNH); P. carnea Butler 1882, holotipo y alotipo examinados, Chile (BMNH); $P$. cinerea Butler 1882, holotipo examinado (hembra), Chile (BMNH); P. squamosa Butler 1882, holotipo examinado (macho), Chile (BMNH); y $P$. turbida Butler 1882, holotipo y alotipo examinado, Chile (BMNH).

Diagnosis: $S$. primata no se puede distinguir de las otras especies por el patrón de coloración, debido a la gran variabilidad de este carácter entre los individuos. El análisis de la armadura genital nos entrega los caracteres que permiten distinguir esta especie de las otras. Los fenotipos anaranjados de machos y hembras pueden ser confundidos con $S$. hyadesi; los fenotipos castaños y salpicados con escamas oscuras se pueden confundir con algunos machos de $S$. distictaria. Las valvas subrectangulares, cucullus con espinas, gnathos en forma de "V"; permiten separar las especies señaladas anteriormente. El ductus bursae está esclerosa- do solo en los bordes inferiores del ostium bursae permite distinguir a las hembras de esta especie.

Redescripción: macho (Fig. 1J): cabeza blanca; frente castaño claro mezclado con escamas castañas y castaño oscuro; antenas pectinadas, del mismo color que las alas; palpos con los dos últimos segmentos de igual longitud, segmento medio tres veces el proximal, color similar a la frente. Tórax y patas del mismo color, siempre más claros que las alas. Abdomen similar en color a las alas posteriores, último segmento rodeado por un conjunto de escamas piliformes. Alas anteriores amarillo pálido; banda antemedial más clara, nace desde la zona basal y llega al último tercio del margen interno; banda medial es siempre más oscura; mancha discal oscura y pequeña; banda postmedial más clara que las demás; banda terminal levemente más oscura que la anterior con zonas más oscuras en la zona media. Alas posteriores similares en color a las alas anteriores, también la cruzan dos bandas oscuras; banda medial recta; banda extramedial ondulada, nace desde el último tercio del margen costal y llega al margen externo.

Hembra (Fig. 1P): similar al macho, presenta una escotadura en la mitad del margen externo de las alas anteriores, dos manchas circulares oscuras pueden o no estar presente; las antenas son simples. Extensión de las alas: 35 $\mathrm{mm}$ a $42 \mathrm{~mm}(\mathrm{n}=45)$.

Genitalia del macho: (Fig. 4B y 4C) uncus subtriangular, con el ápice agudo y esclerosado; gnathos en forma de "V", con el ápice terminado en punta y ornamentado con un conjunto de espinas cónicas; valvas simples, subtriangulares, con un grupo de espinas en el cucullus; dos veces más largas que su ancho máximo; yuxta ornamentada con microespinas, algunas más gruesas en la zona media; saccus subcircular. Aedeagus tubular; vesica armada con dos conjuntos de espinas, el anterior con espinas cortas y gruesas, la posterior con espinas más largas.

Genitalia de la hembra: (Fig. 4A) Bursa copulatrix elongada, corpus bursae globoso y membranoso, con estriaciones longitudinales; ductus bursae esclerosado en la región que bordea el ostium bursae; apófisis posteriores dos veces más largas que las anteriores.

Distribución (Fig. 12): desde la provincia de Valparaíso a la provincia de Magallanes. 

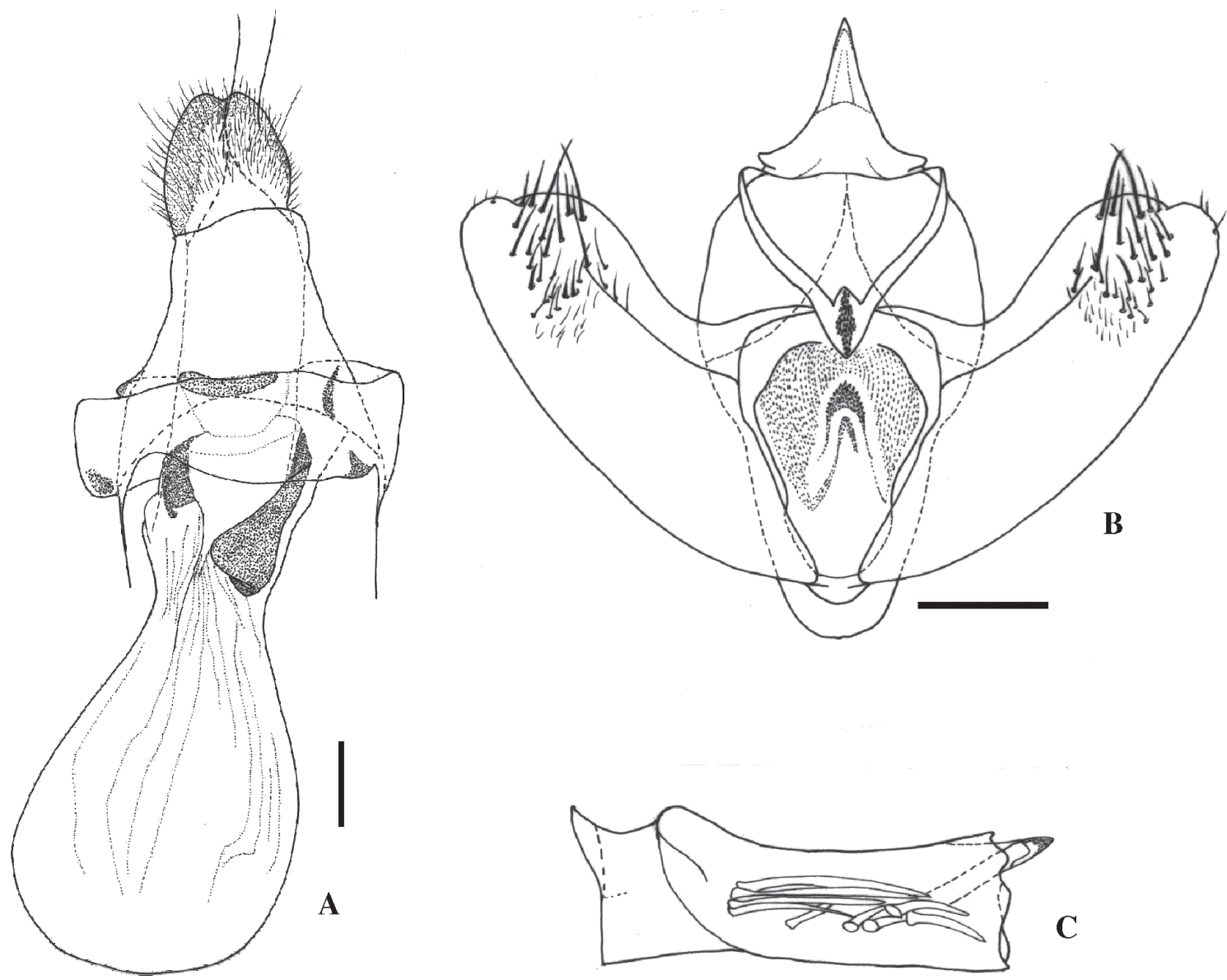

Fig. 4: Syncirsodes primata. (A) genitalia hembra en vista ventral, (B) genitalia macho en vista ventral, y (C) aedeagus en vistal lateral. Escala $1 \mathrm{~mm}$.

Syncirsodes primata. (A) female genitalia in ventral view, (B) male genitalia in ventral view, and (C) aedeagus in lateral view. Scale $1 \mathrm{~mm}$.

Observaciones: Pitkin (2002) propone a $S$. deustata y $S$. primata como dos especies válidas distintas. El análisis de los tipos y sus armaduras genitales nos permiten concluir que $S$. deustata es el sinónimo junior de $S$. primata. Al mismo tiempo, todas las especies descritas en el género Paragonia por Butler (1882), e incorporadas como sinónimos de $S$. deustata por Pitkin (2002), son consideradas sinonimias de $S$. primata, ya que la revisión y comparación de los ejemplares tipos y sus respectivas armaduras genitales así lo sugieren.

Syncirsodes primata es la especie más variable en el patrón de maculación alar, hay ejemplares que se pueden confundir fácilmente con $S$. hyadesi, especialmente aquellas de color amarillento anaranjado. Se pueden encontrar ejemplares amarillentos, sin bandas o con: (1) líneas transversales bien definidas y oscuras. Entre estos, hay individuos con las alas amarillentas abigarradas en toda la superficie por escamas oscuras, líneas transversales bien definida y oscuras, sin bandas perceptibles (Fig.1I); (2) amarillo a castaño claro con la banda medial muy marcada y más oscura, línea oblicua transversal castaño oscuro, dos manchas circulares presentes en la zona media del margen externo, presentes o no (Fig. 1J, 1K, 1L, 1 ̃̃ y 1O); (3) alas castaño claro a castaño ceniciento, banda medial y banda medial marcada y banda antemedial y basal fuertemente perceptible de color castaño oscuro a gris (Fig. 1P); (4) castaño a castaño grisáceo, banda medial perceptible o no, línea oblicua transversal siempre más oscura; (5) rosado ceniciento, banda medial muy notoria y oscura, línea oblicua transversal muy 
notoria; gris claro u oscuro con la banda medial muy marcada y oscura, el espacio que queda entre la línea oblicua transversal y la banda medial de color amarillo pálido (Fig. 1M); (6) gris claro u oscuro, banda medial marcada y oscura, línea oblicua transversal marcada y oscura (Fig. 1Q); (7) alas anteriores bicolor, mancha triangular amarillenta entre la banda medial y posterminal (Fig. 1N). Sin embargo, entre machos $y$ hembras de esta especie no hay diferencias en la maculación alar, como sucede con $S$. distictaria y $S$. hyadesi. S. primata es la especie que tiene la distribución más septentrional del género.

Material examinado: (34 machos, 21 hembras). Chile: holotipo, macho (A. primata) (BMNH); holotipo, hembra ( $P$. deustata) (BMNH); holotipo y alotipo, macho y hembra (P. carnea) (BMNH); holotipo, hembra (P. cinerea) (BMNH); holotipo, macho ( $P$. squamosa) (BMNH); holotipo y alotipo, macho y hembra (P. turbida) (BMNH); Valparaíso: holotipo y alotipo ( $P$. arenosa), Valparaíso, Chile (MBNH); Viña del Mar 1 macho 2-V-1953 (4589); Viña del Mar 1 macho 14-III-1953 (4587) (MNHN); San Antonio: Algarrobo 1 macho 7-1-50 (MZUC). Santiago: La Cisterna 1 macho 1-50 n.n col.; Las Condes 1 macho 8VII-1948. Linares: Principal 1 hembra, II-1889, (MNHN). Cordillera: Pemehue 1 macho (1806) col. Paulsen (MNHN). Maipo: Buin 1 hembra XII-1941, Chile, S. Barros (MZUC); Concepción: Chiguayante, Manquimávida 1 hembra 25-X-51, Hulot col.; S. Pedro 1 hembra 28-X51 Cid col.; Lota 1 macho 5-1-59, Orellana col.; Concepción 1 macho 7-10-76, Angulo col.; 2 machos 14-III-59, 1 macho 22-III-60; 1 macho 31-XII-58; 1 macho 7-IV-60; 1 macho 1-III-61; 1 macho 31-X-59; 1 macho 21-II-59; 1 hembra 21-I-62; 1 hembra 15-VIII-61; 1 hembra $10-X-61 ; 1$ hembra 30-VII-69; 1 hembra 15-IX-60, trampas col. Fototrópica. Hualpén 1 macho 22-10-97; 1 hembra 24-nov.98, T. Ogden col.; 1 macho 31-8-98, P. Bocaz col.; 1 macho 8-12-99, L. E. Parra col.; 1macho emergido 18-11-99; 2 hembras 18-12-00; 1macho emergido 28-10-00; 1 macho emergido 16$11-00 ; 1$ hembra emergida 6-11-00; 1 hembra emergida 23-10-00; 1 hembra emergida 30-1000, P. Bocaz col. (MZUC). Cautín: Cautín 1 hembra Termas de Río Blanco, 3,51(MZUC). Valdivia: Valdivia 1 macho sin datos (4578); 1 macho. Paulsen col.; 1 macho (4600),
(MNHN). Isla Teja Valdivia 1 macho 20 -0886, D. Jackson col. MZUC); Nilahue 1 macho 4-1-50 (MZUC); 1 macho sin datos col. Paulsen (5313) (MNHN). Llanquihue: Maullín 1 hembra II-1-1943 (MZUC). Magallanes: Tres Puentes 1 macho Dic-1952 Rodríguez col. (MZUC).

Syncirsodes straminea Butler 1882 (Fig. 1R-T)

Syncirsodes straminea Butler 1882: 343; Angulo y Casanueva 1981: 18; Pitkin 2002: 318.

Material tipo: Syncirsodes straminea Butler 1882, sintipo examinado (macho) (MNHN), Chile.

Diagnosis: S. straminea se distingue fácilmente de las otras especies por su patrón de coloración amarillo pálido. Sin embargo, el macho puede ser confundido con machos amarillos de $S$. hyadesi. La genitalia del macho se distingue porque las valvas son subtriangulares y el ductus bursae es pentagonal y esclerosado en la genitalia de la hembra.

Redescripción: Macho (Fig. 1S): cabeza y frente blanca; antenas pectinadas ocre; palpos similar en color a las antenas, segmento medio tres veces más largo que el proximal; tórax y patas ocre, con algunas escamas castañas dispersas en la superficie. Abdomen del mismo color que las alas anteriores. Alas anteriores amarillo pálidas; banda postmedial y adterminal de un tono más claro que la banda medial; banda medial delimitada en ambos extremos por líneas ocres; banda antemedial similar en color a la banda postmedial; mancha discal oscura y muy pequeña. Alas posteriores, con color levemente más pálido que las alas anteriores; línea extradiscal imperceptible; mancha discal, oscura y muy pequeña.

Hembra (Fig. 1R): similar al macho. Se distingue de este porque las alas anteriores presentan una escotadura justo en la mitad del margen externo; antenas simples. Extensión de las alas: 37 a $40 \mathrm{~mm}(\mathrm{n}=11)$.

Genitalia del macho (Fig. 5B y 5C): uncus subtriangular, ápice levemente esclerosado terminado en una aguda punta; gnathos en forma de $\mathrm{U}$, ápice terminado en una punta corta y aguda, con un conjunto de pequeñas espinas cónicas; yuxta ornamentada con microespinas; socius ausente; valvas subtriangulares, cucullus con un conjunto de cerdas pequeñas y escasas, saccus subcircular. Aedeagus tubular; vesica 
armada con dos conjuntos de espinas cónicas, las posteriores siempre más largas que las anteriores.

Genitalia de la hembra (Fig. 5A): bursa copulatrix elongada, corpus bursae con la región anterior globosa y membranosa, la región posterior esclerosada y con estrías longitudinales; signum ausente; ductus bursae esclerosado en el centro, de forma pentagonal; apófisis posteriores dos veces más largas que las anteriores.

Distribución (Fig. 12): desde la provincia de La Araucanía hasta la provincia de Magallanes.

Observaciones: $S$. straminea es la especie más fácil de distinguir por la coloración, ya que presenta menor variación entre los machos del género, en tanto las hembras varían entre dos tonalidades: amarillo pálido o castaño rojizo (Fig. 1T).

Material examinado: (6 machos, 7 hembras): Chile: sintipo, macho (MNHN). Araucanía: 1 hembra febrero 88 col. Paulsen (4614)
(MNHN), 1 hembra II. 52 (4617) (MNHN); Cautín: Termas de Río Blanco 4 machos 3. 51 (MZUC), 5 hembras 3. 51 (MZUC); Magallanes: Tierra del Fuego 1 macho, diciembre 1990 Venegas col. (MZUC).

\section{DESCRIPCIÓN DE LOS ESTADOS INMADUROS}

Syncirsodes distictaria (Mabille 1885) (Fig. 6G-6L, Fig. 7A y Fig. 10).

Huevos (Fig. 6G-6J): esféricos, color amarillo pálido; zona micropilar achatada, micropila con 10 poros micropilares; celdas primarias y secundarias con apariencia reticulada y en posición radial; exocorion con ornamentaciones también reticuladas; aeropilas circulares, formando costillas que llegan solo al primer tercio de la superficie lateral del huevo.
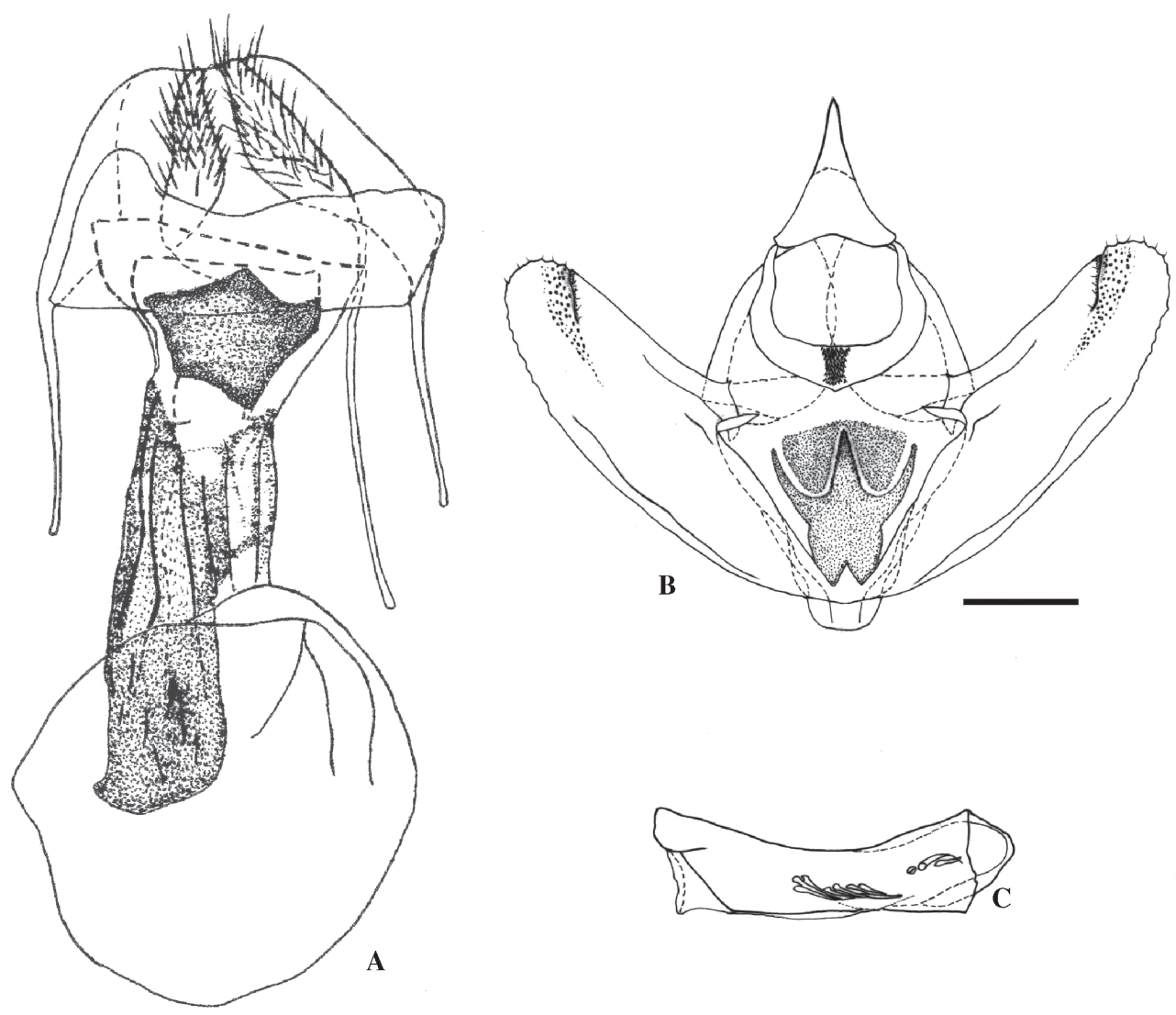

Fig. 5: Syncirsodes straminea. (A) genitalia hembra en vista ventral, (B) genitalia macho en vista ventral, y $(\mathrm{C})$ aedeagus en vista lateral. Escala $1 \mathrm{~mm}$.

Syncirsodes straminea. (A) female genitalia in ventral view, (B) male genitalia in ventral view, and (C) aedeagus in lateral view. Scale $1 \mathrm{~mm}$ 

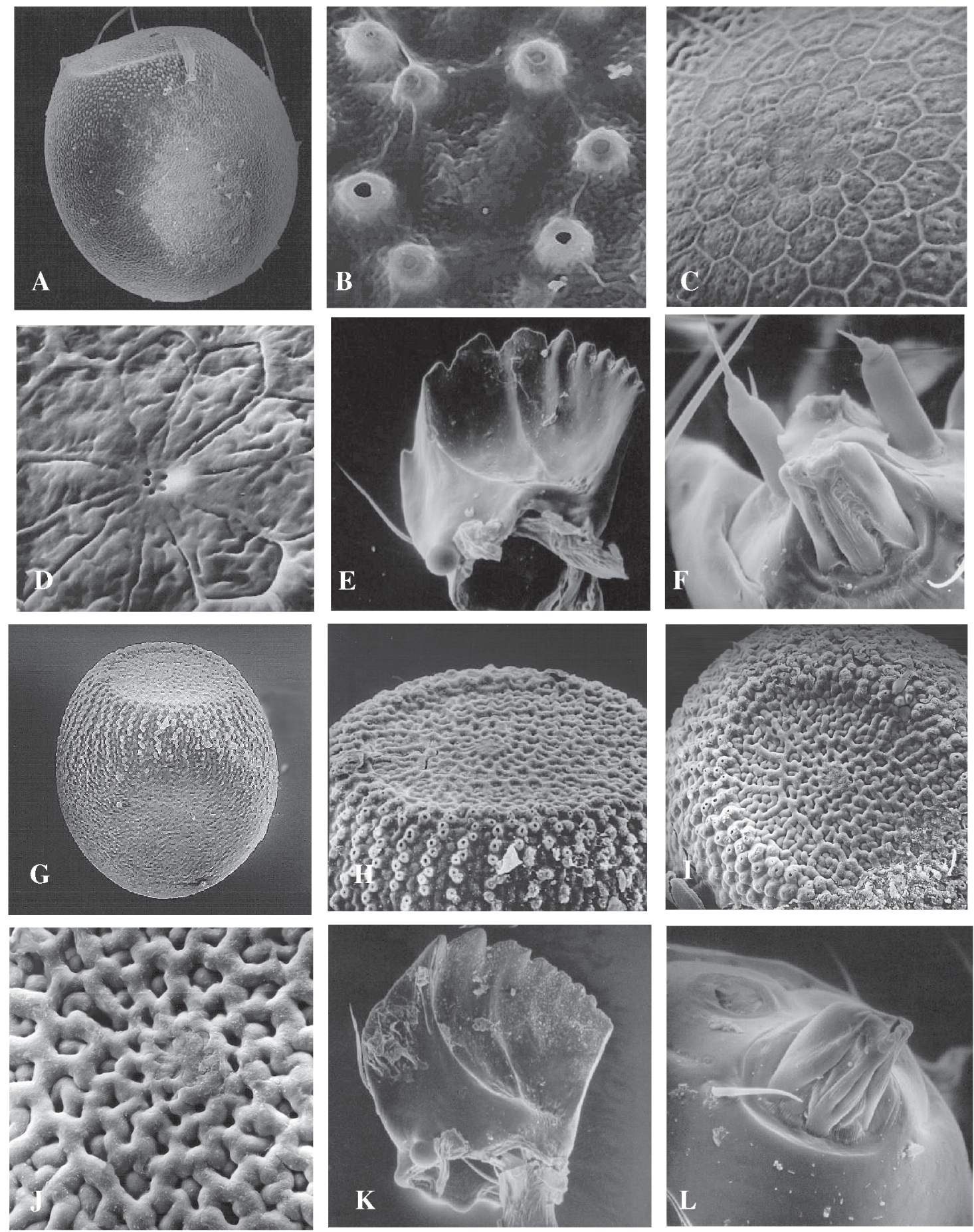

Fig. 6: Syncirsodes primata: (A) huevo en vista lateral, (B) aerópilas, (C) poros micropilares y celdas primarias, (D) zona micropilar (E) mandíbula de larva en vista ventral y (F) espinerete. $S$. distictaria: $(\mathrm{G})$ vista lateral huevo, $(\mathrm{H})$ aerópilas, (I) zona micropilar, (J) área micropilar y celdas primarias, $(\mathrm{K})$ vista ventral mandíbula derecha en larva de último estadio, y $(\mathrm{L})$ espinerete. Escala: (A, G y H) $80 \mu \mathrm{m}$; (E) $90 \mu \mathrm{m}$; (D, F, H, I y L) $400 \mu \mathrm{m}$, y (B, C y J) $1.600 \mu \mathrm{m}$.

Syncirsodes primata: (A) egg in lateral view, (B) aeropyles, (C) micropylar pores and primary cells, (D) micropylar area, (E) larva jaw in ventral view and (F) spinneret. S. distictaria: (G) egg in lateral view, (H) aeropyles; (I) micropylar area, (J) micropylar pores and primary cells, $(\mathrm{K})$ mandible larva in ventral view, and (L) spinneret. Scale: (A, G y K) $80 \mu \mathrm{m}$; (E) $90 \mu \mathrm{m} ;(\mathrm{D}, \mathrm{F}, \mathrm{H}, \mathrm{I}$ y L) $400 \mu \mathrm{m}$, and (B, C y J) $1,600 \mu \mathrm{m}$. 

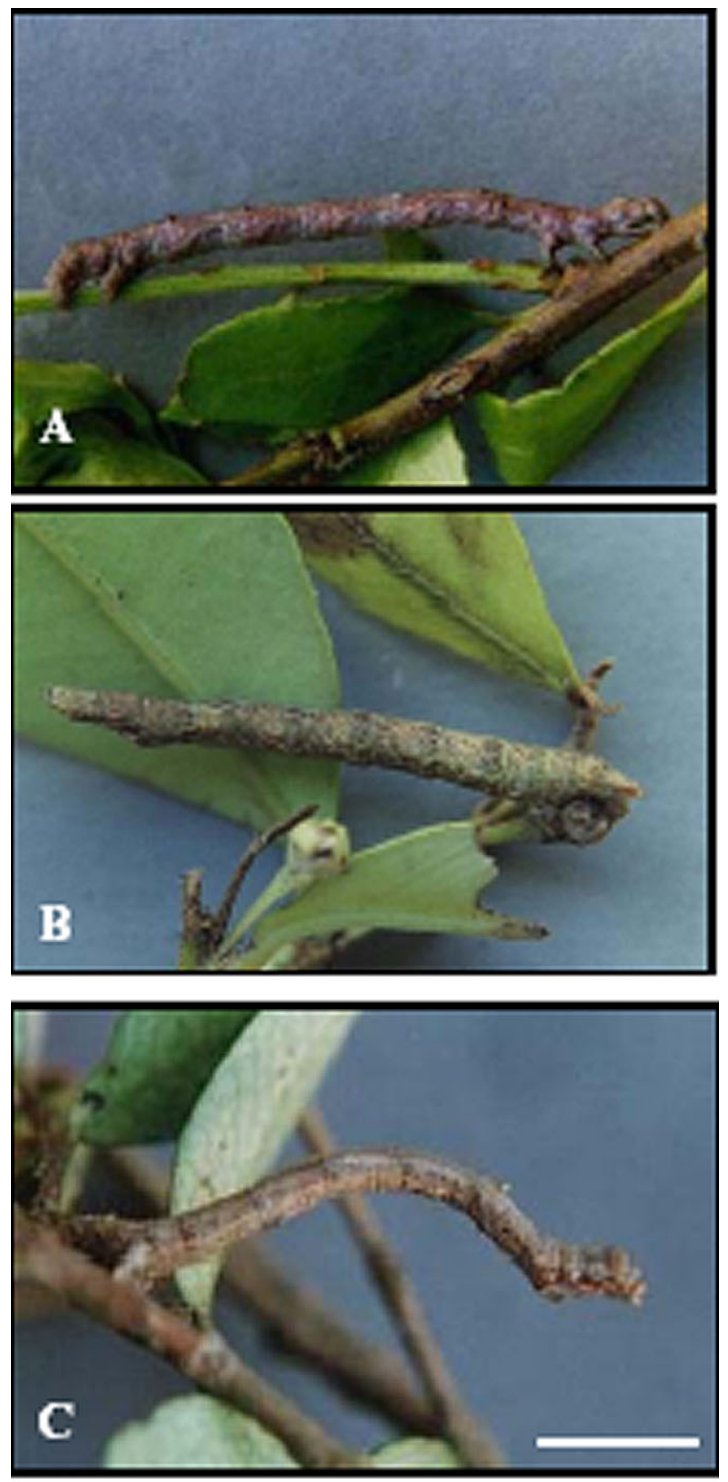

Fig. 7: (A) Vista general de larva de S. distictaria sobre Laurelia sermpervirens. (B y C) Larvas S. primata. (B) Larva de último estadio sobre Myrceugenia planipes; (C) larva de último estadio sobre Myrceugenia obtusa. Escala $1 \mathrm{~cm}$.

(A) General view of $S$. distictaria larva on Laurelia sermpervirens. (B and C) S. primata larvae. (B) Last instar larva on Myrceugenia planipes; (C) last instar larva on Myrceugenia obtusa. Scale $1 \mathrm{~cm}$.

Larva (Fig. 7A): cuerpo delgado y elongado rosado oscuro, con muchas líneas cortas y negras de forma irregular; cerdas de color negro, cortas y finas; cerdas dorsales nacen de pinaculas en los segmentos abdominales uno, cuarto, quinto y octavo. Cabeza hipognata, redondeada y deprimida. Mandíbulas subcuadrangulares con siete dientes, cinco de ellos bicúspides, una seta en el margen externo (Fig. 6K), espinerete tubular (Fig. 6L). Cuerpo largo y delgado, aproximadamente $50 \mathrm{~mm}$ de largo en el último estadio $(n=1)$, más ancho en la zona posterior y aguzado en la anterior, el tercer segmento torácico presenta un tubérculo desde donde nace el tercer par de patas. Por falta de material no se pudo analizar la quetotaxia. Estadios larvales indeterminados, ya que se trabajó solo con un ejemplar, que correspondió a un estado de desarrollo larval intermedio.

Pupa (Fig. 10): obtecta, amarilla pálida con puntos negros en el nacimiento de las cerdas, largo $19 \mathrm{~mm}$ y ancho $6 \mathrm{~mm}(\mathrm{n}=1)$. Vista ventral (Fig. 10A): ojos circulares; clípeo-labro plano con dos puntos en el centro; podoteca 1 alcanza el segundo tercio del tercer segmento abdominal; espiritrompa alcanza el segundo tercio del cuarto segmento abdominal; podoteca 2 y antenas alcanzan el último tercio del cuarto segmento abdominal. Vista lateral (Fig. 10B): con escotadura en el noveno segmento abdominal. Vista dorsal (Fig. 10C): en los segmentos torácicos presenta puntos negros desde donde nacen cerdas oscuras; cremaster con dos ganchos medianamente largos y gruesos que nacen de la base del décimo segmento, tres pares más pequeños y delgados nacen del último tercio del mismo segmento (Fig. 10D-10F).

Material examinado: huevos: obtenidos de hembra emergida el 11-11-00 (MZUC). Larvas: 1 larva y 2 cápsulas cefálicas, Hualpén 1 exuvia y cápsula cefálica colectada en 11-10-00, P. Bocaz col.; 1 cápsula cefálica 29-9-00, P. Bocaz col. (MZUC). Pupa: 1 pupa Hualpén 11-1000, P. Bocaz col. (MZUC).

Syncirsodes primata (Walker, 1862). (Fig. 6 A$D ; 7 B-C ; 11)$

Huevo (Fig. 6A-6D): esféricos, verdes; zona micropilar achatada; micropila con 10 celdas primarias romboidales, las secundarias hexagonales; aerópilas tubulares (Fig. 6B), se ubican en el borde del área micropilar; exocorion sin ornamentaciones.

Larva (Fig. 7B y 7C) de S. primata tiene cuerpo alargado y delgado, la zona posterior más gruesa que la anterior, con gran variación de color, que van desde un verde claro a castaño, y también se detectó variación morfológica, por la 
presencia y ausencia de tubérculos dorsales en los segmentos abdominales (Bocaz et al. 2003), cabeza (Fig. 8A y 8B), hipognata cuadrangular y aplanada, labro con cuatro pares de cerdas, tercer segmento torácico con un tubérculo de donde nace el tercer par de patas; espiráculos anaranjados. Mandíbulas subcuadrangulares con 10 dientes de forma puntiaguda irregular (Fig. 6E), espinerete tubular (Fig. 6F). Quetotaxia (Fig. 9): cerdas dorsales (D) bisetosa en todos los segmentos abdominales, excepto en A9; cerdas extradorsales (XD) bisetosas, presentes solo en el primer segmento torácico; cerdas subdorsales (SD) bisetosas en el primer, segundo y tercer segmento torácico, también en el décimo segmento abdominal, unisetosa en los demás segmentos; cerdas laterales (L) bisetosa en el primer segmento torácico, trisetosas en los segmentos abdominales, unisetosa en A9; cerdas subventrales (SV) bisetosas en los segmentos torácicos y $\mathrm{A} 10$, unisetosa en $\mathrm{A} 7, \mathrm{~A} 8$ y $\mathrm{A} 9$, cuádruple en A6 sobre el espuripedio; cerdas ventrales (V) unisetosas en todos los segmentos toráxicos y en A6, A8 y A9, bisetosa en A1-A5 y en A10. Espuripedios solo en el sexto y décimo segmento abdominal; crochets uniordinales, biseriales con mesoserie interrumpida. La larva presenta siete estadios, que fueron obtenidos de las distintas mudas de una misma larva.

Pupa: obtecta (Fig. 11), castaño claro con espiráculos y cerdas más oscuras, largo promedio $16,1 \mathrm{~mm}$ y ancho promedio $4,5 \mathrm{~mm}$. ( $\mathrm{n}=$ 7). Vista ventral (Fig. 11A): ojos cuadrangulares; clípeo-labro semicircular; podoteca 1, alcanza el tercer segmento abdominal; espiritrompa, podoteca 2 y ceratoteca, alcanzan el cuarto segmento abdominal, décimo segmento estriado. Vista lateral (Fig 11B): escotadura lateral en el noveno segmento abdominal. Vista dorsal (Fig. $11 \mathrm{C}$ ): con hendidura entre octavo y noveno segmento abdominal; cremaster (Fig. $11 \mathrm{D}-11 \mathrm{~F})$ con dos espinas centrales gruesas, dos pares en los costados del décimo segmento abdominal, más pequeñas y delgadas.

Material examinado: huevos: obtenidos de hembras emergidas en laboratorio durante los meses de octubre a diciembre del 2000 y enero del $2001 \mathrm{y}$, de una hembra grávida Hualpén 810-00, P. Bocaz col. (MZUC). Larvas: 15 larvas, 3 exuvias. Hualpén 10 larvas 8-10-99, L. E. Parra col.; 1 larva 18-11-00; 1 larva 6-9-00; 1 larva 16-10-00; 2 larva 30-10-00; P. Bocaz col. 1 exuvia $7-10-00 ; 1$ exuvia $22-10-00 ; 1$ exuvia 28-11-00, P. Bocaz col. (MZUC). Pupas: 7 pupas obtenidas en laboratorio.

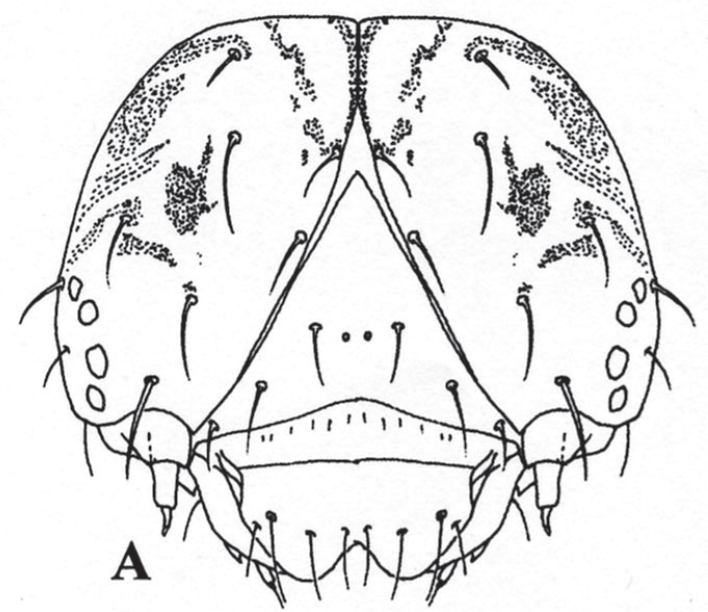

B

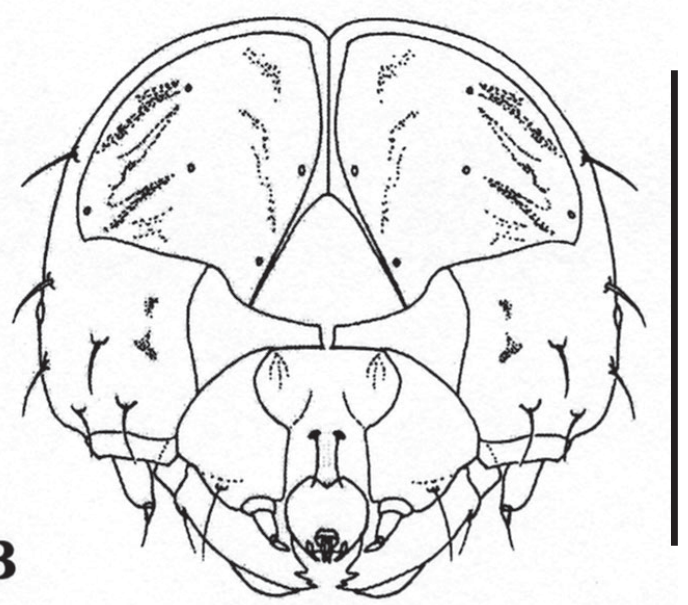

Fig. 8: Syncirsodes primata. Cápsula cefálica del último estadio larval de; (A) vista dorsal, (B) vista dorsal. Escala $1 \mathrm{~mm}$.

Syncirsodes primata. Head capsule of the last instar larval; (A) dorsal view, (B) dorsal view. Scale $1 \mathrm{~mm}$.

\section{Bionomía}

La larva de $S$. distictaria (Mabille 1885) fue encontrada de último estadio a fines de septiembre, comiendo las hojas nuevas de Laurelia sempervirens ( $\mathrm{R}$ et $\mathrm{P}$.) Tul. (Monimiaceae). El color rosado oscuro que presenta la larva concuerda con la zona de los tallos inferiores de la planta donde fue colectada, sí era muy similar en forma y color a la larva de $S$. primata que se alimenta de la misma planta. Durante la fase prepupal, la oruga baja su actividad, sus movimientos son lentos y deja de comer. La pupa- 
ción tiene lugar dentro de un capullo que la propia larva teje, entre dos hojas que junta con su seda. El periodo pupal se completa en 30 días en la única larva analizada. La emergencia del adulto se produjo en noviembre.

El ciclo de vida es univoltino con periodo de vuelo entre noviembre y fines de marzo (Fig. 13). Las larvas de S. primata fueron encontradas durante septiembre hasta fines de noviembre, en distintas plantas hospederas, en Aextoxicon punctatum R. et P., Cryptocaria alba (Mol.) Looser, Laurelia sempervirens (R.et P.) Tul., Lithrea caustica (Mol.) H. et A., Luma apiculata (DC) Burret, Myrceugernia planipes (H. et A.) Berg, Myrceugenia obtusa, Peumus boldus Mol., Rhaphithamnus spinosus (A. L. Juss), Aristotelia chilensis (Mol.) Stuntz y Ribes punctatum R. et. P. Al eclosionar del huevo, la larva tiene el dorso y vientre negro, una línea lateral blanca recorre todo el cuerpo desde el último segmento abdominal al primer segmento torácico. Este patrón se mantiene hasta el cuarto estadio, luego la coloración se hace uniforme siempre más clara; posteriormente adquieren un diseño cromático característico entre las distintas variedades. La morfología y color de la oruga le permite camuflarse con los tallos de cada una de las plantas hospederas. En A. punctatum la coloración del estado larval es castaño rojizo, en $C$. alba la larva es de color oscuro en estadios tempranos y en los estadios avanzados van desde rosado oscuro al castaño verdoso; en $M$. planipes ocre claro; en
L apiculata castaño oscuro a castaño claro; en $L$. caustica verde grisáceo; en $L$. sempervirens rosado claro a rosado oscuro; en $P$. boldus verde amarillento; en $R$. spinosus es castaño claro a grisáceo. El periodo larval se extiende entre mediados de septiembre a fines de noviembre. Las larvas comen las hojas nuevas de cada especie de planta hospedera. La etapa prepupal observada duró entre 48 y $72 \mathrm{~h}$, periodo en el cual la larva deja de comer, su cuerpo se ensancha y su longitud se reduce, con los escasos movimientos que logra realizar une los extremos de una o dos hojas, teje un capullo muy simple y pupa. El periodo pupal es muy relativo, va desde los 15 a 24 días.

El ciclo de vida de $S$. primata es univoltino y la época de vuelo va desde fines de octubre hasta mediados de marzo (Fig. 14).

\section{DISCUSIÓN}

Todas las especies incluidas en Syncirsodes fueron previamente incorporadas al género $E n$ nada por Scoble (1999). Pitkin (2002) restringe el género Ennada a la especie tipo, E. flavaria Blanchard 1852. De esta especie solo se conoce la hembra, y a diferencia de las hembras de Syncirsodes, las alas anteriores no tienen la escotadura característica del margen externo. Además, la genitalia tiene un signum en forma de espina condición ausente en las especies de Syncirsodes, y que se constituye en la autapo-

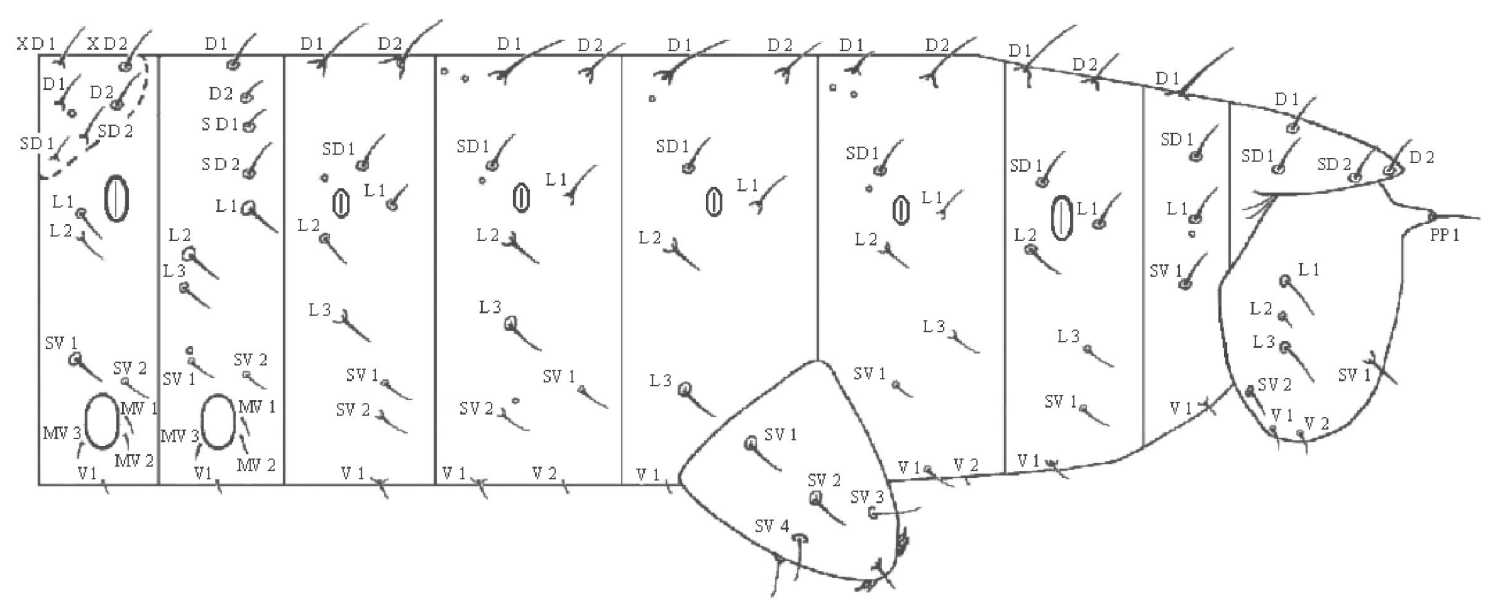

Fig. 9: Quetotaxia larval de Syncirsodes primata.

Syncirsodes primata larval chaetotaxy. 
morfia que define Ennada (Pitkin 2002). Por último, hay evidencia morfológica (patrón de venación de las alas posteriores) con la que $E$. flavaria podría se incorporada a la subfamilia Larentiinae, a pesar de la carencia de las bandas transversales en las alas anteriores (Pitkin 2002).

El género Syncirsodes queda conformado por las siguientes especies: $S$. distictaria (Mabille 1885); S. primata (Walker 1862), S. hyadesi (Mabille 1885), S. straminea Butler 1882 y S. subornata (Walker 1863). Syncirsodes subornata (Walker 1863) es incluida en el género por Pitkin (2002) cuya localidad tipo es Uruguay. El material tipo de esta especie está representado solamente por una hembra de la cual no se tiene la armadura genital (perdida). El ejemplar es muy parecido a la hembra de $S$. distictaria en su patrón de maculación (Fig. $1 D$ ); además posee la escotadura en las alas anteriores rasgo característico del género. Basándose en estos caracteres y siguiendo a Pitkin (2002) hemos decidido dejarla como una especie válida del género Syncirsodes. La revisión futura de otros ejemplares y sus armaduras genitales permitirá llegar a conclusiones más claras respecto a esta especie. Por otro lado, la comparación morfológica de las armaduras genitales de los tipos de las especies $S$. deustata y $S$. primata, nos permite concluir que el ejemplar macho del tipo de primata corresponde al macho del tipo de $S$. deustata, y que la hembra tipo de primata es verdaderamente $S$. distictaria. Por lo tanto, la especie $S$. primata (Walker 1862) es el sinónimo senior de S. deustata (Felder \& Rohgenhofer 1875).

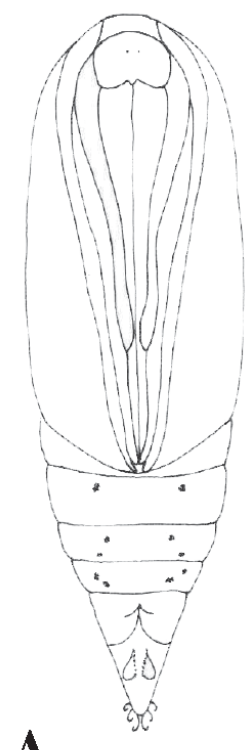

A

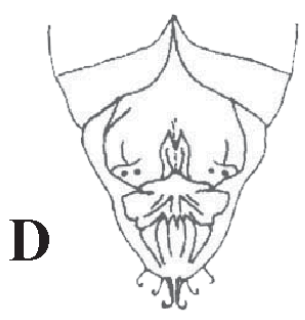

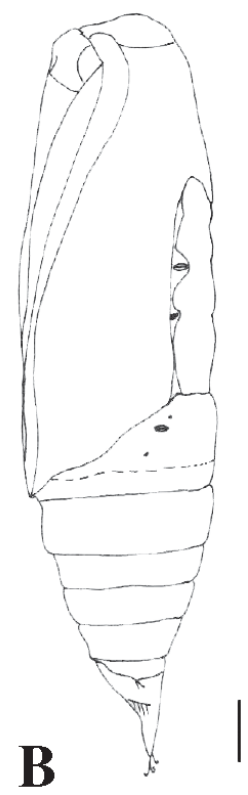

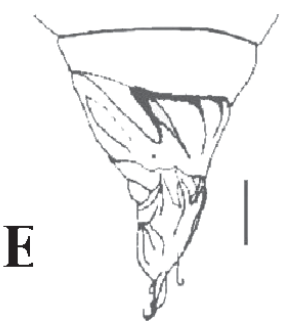

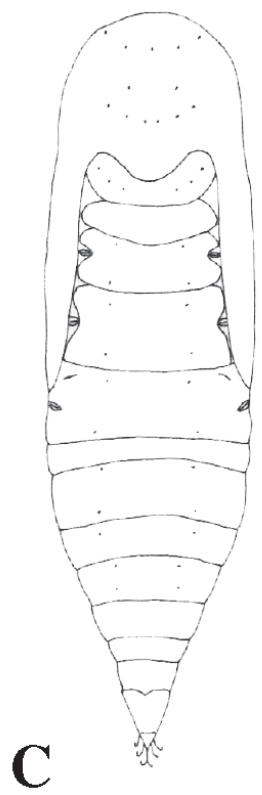

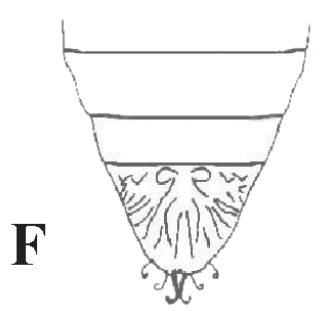

Fig. 10: Pupa Syncirsodes distictaria. (A) Vista ventral, (B) vista lateral y (C) vista dorsal; (D-F) terminalia pupa, (D) vista ventral, (E) vista lateral y (F) vista dorsal. Escala $1 \mathrm{~mm}$. 

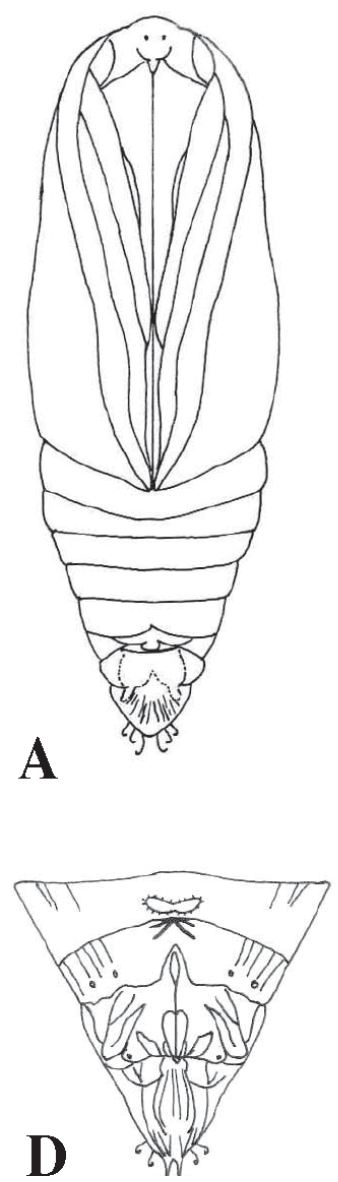
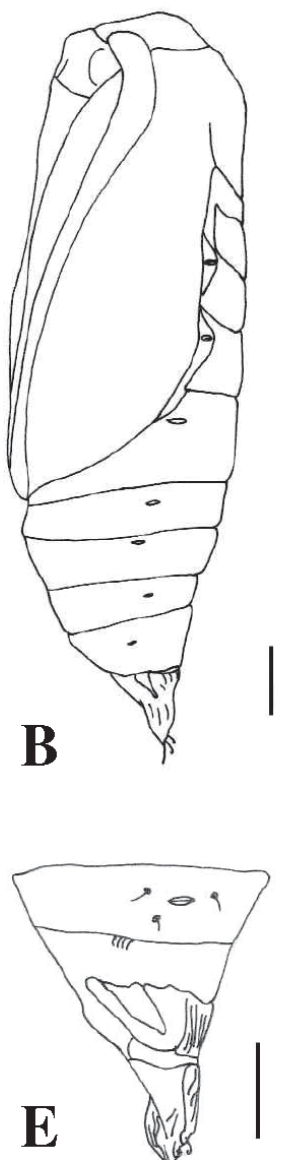
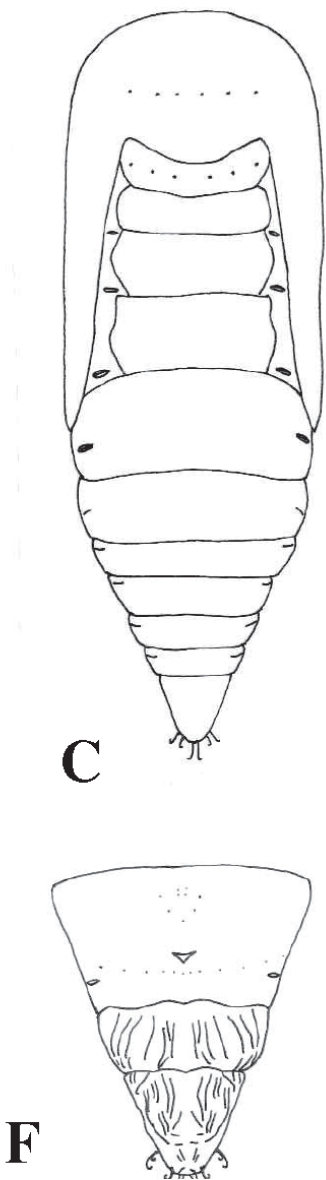

Fig. 11: Pupa Syncirsodes primata. (A) Vista ventral, (B) vista lateral y (C) vista dorsal; (D-F) terminalia pupa, (D) vista ventral, (E) vista lateral, y (F) vista dorsal. Escala $1 \mathrm{~mm}$.

Syncirsodes primata pupa. (A) Ventral view, (B) lateral view and (C) dorsal view; (D-F) end of the pupa, (D) ventral view, (E) lateral view, and (F) dorsal view. Scale $1 \mathrm{~mm}$.

Las especies incluidas en Syncirsodes parecen estar relacionadas en la forma y color de las alas. La principal característica de este género es su gran variabilidad interespecífica e intraespecífica en la coloración y patrón de maculación de las alas anteriores, condición que no permite discriminar la pertenencia de individuos a una determinada especie. Los machos de $S$. primata, $S$. hyadesi y $S$ distictaria comparten muchas de las características de su maculación. Los machos de $S$. straminea se confunden fácilmente con los machos de $S$. hyadesi cuando la coloración coincide. Las hembras de $S$. hyadesi y S. straminea mantienen un patrón de maculación consistente, por el contrario las variedades anaranjadas de $S$. primata y $S$. hyadesi se confunden fácilmente. El análisis de la armadura genital de machos y hembras, permite sepa- rar cada una de las especies que conforman el género Syncirsodes, asegurando una correcta identificación de los ejemplares. El valor diagnóstico de las armaduras genitales en Lepidoptera ha sido ampliamente aceptada y documentada por diversos autores (Scoble 1986, Choi 1999). Estas diferencias en la morfología genital (estructuras esclerosadas) son las precursoras y forman parte de los mecanismos precigóticos que impiden la hibridación entre las diferentes especies.

El conocimiento de los estados inmaduros, nos ayudan a comprender la biología de las diferentes especies; asimismo, sus rasgos morfológicos y de comportamiento nos entregan antecedentes que pueden ser utilizados para comprender la filogenia y evolución del género y del grupo (características morfológicas de la 
larva, huevo, comportamiento y hábitos alimentarios), pues estos caracteres clarifican las incongruencias de los esquemas de clasificación propuestos para insectos adultos (Zakvatkin 1969, Jerez 1996).

Solamente se conoce en forma completa la biología de las especies $S$. distictaria y $S$. primata. Los ciclos de vida de ambas especies concuerdan con los descritos para esta familia, y para las demás especies que habitan la Península de Hualpén (Bocaz 2001). Morfológicamente, cuando se comparan las larvas de ambas especies, estas reflejan muchos caracteres comunes que dificultan su separación. Sin embargo, las mandíbulas presentan diferencias en el número y posición de los dientes.

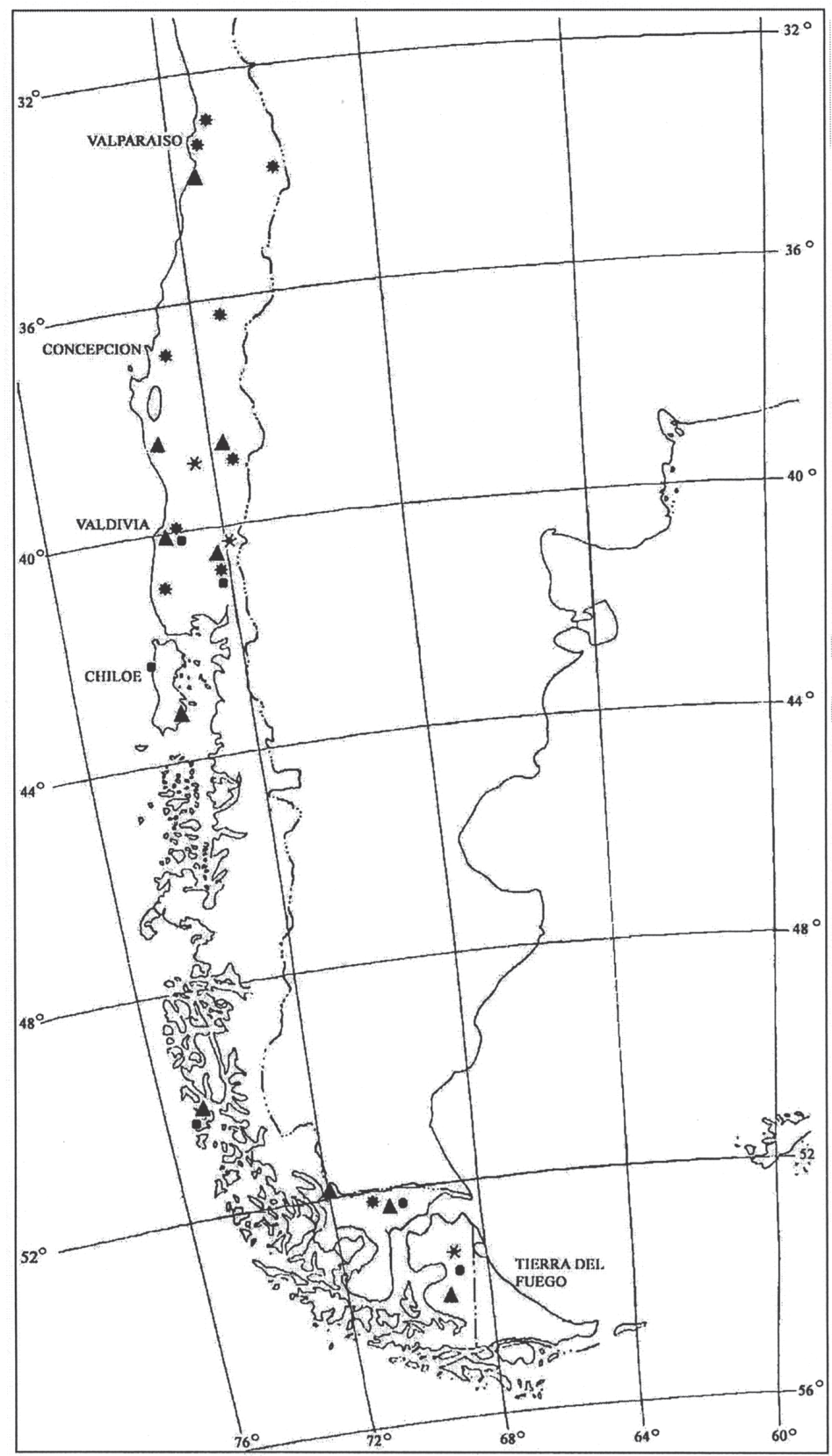

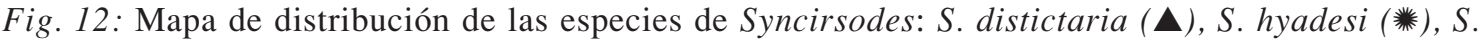
primata, S. straminea $(*)$.

Distribution map of the Syncirsodes species: S. distictaria ( $\mathbf{\Delta})$, S. hyadesi (*), S. primata, S. straminea (*). 


\begin{tabular}{|c|c|c|c|c|c|c|c|c|c|c|c|c|}
\hline Huevo & & & & & & & & & & & $=$ & \\
\hline Larva & & & & & & & & & & & & \\
\hline Pupa & & & & & & & & & & & & \\
\hline Adulto & & & & & & & & & & & & \\
\hline & Ene & Feb & Mar & Abr & May & Jun & Jul & Ago & Sep & Oct & Nov & Dic \\
\hline
\end{tabular}

Estimado:

Con datos:

Fig. 13: Ciclo de vida de Syncirsodes distictaria.

Life cycle of Syncirsodes distictaria.

\begin{tabular}{|l|l|l|l|l|l|l|l|l|l|l|l|l|}
\hline Huevo & & & & & & & & & & & & \\
\hline & \hline Larva & & & & & & & & & & & \\
\hline
\end{tabular}

Estimado:

Con datos:

Fig. 14: Ciclo de vida de Syncirsodes primata.

Life cycle of Syncirsodes primata.

La larva de $S$. primata, presenta gran variabilidad fenotípica en color y forma, siendo esto congruente con la variabilidad fenotípica de los adultos y con la gran variedad de plantas hospederas a las que está asociada (Bocaz et al. 2003). La larva de $S$. primata presenta hábitos polífagos en especies de plantas hospederas asociadas al bosque costero de la VIII Región (península de Hualpén), esclerófilo e higrófilo en ausencia de Nothofagus (Polymeris 1995). También se la ha encontrado en Roble (Notho- fagus obliqua) y en Quillay (Quillaja saponaria) (Urra com. per.) en localidades más septentrionales de Chile central.

Los datos sobre bionomía nos permiten comprender la dinámica de estas especies dentro de su hábitat y la relación existente con la vegetación. La biología de las especies aquí mencionadas, da luz de la estrecha asociación con las plantas hospederas, donde se desarrolla toda la etapa preimaginal. El ciclo de vida de $S$. distictaria y $S$. primata se caracteriza por la 
coincidencia entre el período larval y crecimiento vegetativo de las plantas (ajuste fenológicos), ya que las larvas se alimentan de las hojas que emergen en la estación primaveral.

La distribución abarca las regiones Andina y Neotropical (Morrone 2001). El género tiene una amplia distribución en nuestro país, S. primata es la especie que muestra mayor rango de distribución, desde Coquimbo hasta Magallanes. La especie con un menor rango de distribución es $S$. straminea, solo se ha encontrado desde la Novena a la Duodécima Región. Syncirsodes subornata es la única especie con distribución Neotropical, en la provincia Pampa propuesta por Morrone (2001).

\section{AGRADECIMIENTOS}

Los autores agradecen a Ariel Cammouseight y Mario Elgueta (MNHN), Vivianne Jerez (MZUC) Chile y Fernando Navarro y Adriana Chalup (IML) Argentina, por la valiosa cooperación en la obtención de los ejemplares tipos y literatura relacionada con este estudio. A Malcolm J. Scoble (BMNH) por las facilidades otorgadas durante la visita de Luis E. Parra al The Natural History Museum y el estudio de los tipos depositados en aquella institución. A Linda Pitkin por su valiosa ayuda en la revisión a posteriori de los tipos depositados en BMNH. A Alicia Marticorena por su ayuda en la identificación de plantas hospederas y a Héctor A. Vargas, Enrique Mundaca, Manuel Ojeda y T. Heath Ogden por su colaboración durante los muestreos. Agradecemos especialmente a César Soto Torres por el mejoramiento de las fotografías. Al laboratorio de Microscopía Electrónica de la Universidad de Concepción y su personal Raúl Alarcón, Julio Pugin y Hugo Pacheco, por las fotografías de microestructuras incluidas en este trabajo. Este trabajo contó con el apoyo financiero de los Proyectos 98.113.047-1.D.1, 200.113.056-1.0 y el "Proyecto Instrumental Científico 2001" de la Dirección de Investigación de la Universidad de Concepción.

\section{LITERATURA CITADA}

ARTIGAS JN (1994) Entomología económica. Volumen 2. Ediciones Universidad de Concepción, Concepción, Chile. 943 pp

ANGULO AO \& ME CASANUEVA (1981) Catálogo de los lepidópteros geométridos de Chile (Lepidoptera:
Geometridae). Boletín de la Sociedad de Biología de Concepción (Chile) 51: 7-39.

BOCAZ PA. (2001) Taxonomía e historia natural de los geométridos de la península de Hualpén VIII Región. Tesis para optar al grado de Licenciado en Educación, Universidad de Concepción, Concepción, Chile. 100 pp.

BOCAZ PA, LE PARRA \& P VICTORIANO (2003) Larval morphological variation and its relation to host plants in Syncirsodes primata (Lepidoptera: Geometridae). Gayana Zoología (Chile) 67: 39-44.

BUTLER AG (1882) Hetrocerous Lepidoptera collected in Chili by Thomas Edmonds, Esq. Part III. Geometrites. Transactions of the Entomological Society of London: 339-423, plate 16 .

BUTLER AG (1893) On small collection of Lepidoptera from Chili. The Annals and Magazine of Natural History series 6, 12: 457-467.

CHOI SW (1999) Taxonomic review of a new genus, Diathera gen. n., from Southeast Asia (Lepidoptera, Geometridae: Larentiinae). Journal of Natural History 33: 1039-1048.

FELDER C \& AF ROGENHOFER (1875) Reise der österreichischen Fregatte Novara um die Erde (Zoologischer Theil) Band 2 (Abtheilung 2): pls. 121-140. Weine, Austria.

HEPPNER JB (1991) Faunal regions and the diversity of Lepidoptera. Tropical Lepidoptera (Supplement 1) 2: $1-85$.

JEREZ V (1996) Biology and phylogenetic remarks of the subantarctic genera Hornius, Stenomella and Dictyneis (Chrysomelidae-Eumolpinae). 239-258. En: Jolivet P, M Cox \& Petitperret (eds) Chrysomelidae biology. Volume 3. General Studies, Kluwer Academic Publishers, The Netherlands. 365 pp.

KLOTS AB (1970) Lepidoptera. En: Tuxen SL (ed) Taxonomist's glossary of genitalia in insects: 115130. Second edition. SHSA, New York, New York, USA.

MABILLE MP (1885) Diagnoses de Lépidoptères nouveaux. Bulletin de la Societé Philomathique Paris (France) 9: 55-70.

MORRONE JJ (2001) Biogeografía de América Latina y el Caribe. M\&T Manuales y Tesis SEA 3: 1-148.

PARRA LE (1991) Revisión y filogenia del género Pachrophylla Blanchard, 1852 (Sensu auctorum) (Geometridae: Larentiinae: Trichopterygini). Gayana Zoología (Chile) 55: 145-199.

PARRA LE (1995) Lepidoptera. En: Simonetti JA, MTK Arroyo, AE Spotorno \& E Lozada (eds) Diversidad biológica de Chile: 269-279. Comisión Nacional de Ciencia y Tecnología, Santiago, Chile. 363 pp.

PARRA LE (1999a) Revision of the Neotropical genus Psilaspilates (Lepidoptera: Geometridae). Entomological Society of America 92: 460-472.

PARRA LE (1999b) Revisión del género Euclidiodes Warren, 1895 (Lepidoptera: Geometridae). Revista Chilena de Historia Natural 72: 643-659.

PARRA LE \& H VARGAS (2000) Revisión del género Neorumia Bartlett-Calvert, 1893 (Lepidoptera: Geometridae). Revista Chilena de Entomología 27: 91-98.

PITKIN LM (2002) Neotropical ennomine moths: a review of the genera (Lepidoptera: Geometridae). Zoological Journal of the Linnean Society 135: 121-401.

POLYMERIS C (1995) Vegetación actual de la península de Hualpén: clasificación y dinámica. Tesis para optar al grado de Magíster en Ciencias con mención 
en Botánica, Universidad de Concepción, Concepción, Chile. 190 pp.

SALKELD EH (1983) A catologue of the eggs of some Canadian Geometridae (Lepidoptera), with comments. Memoirs of the Enthomological Society of Canada 126: 1-271.

SCOBLE MJ (1986) The structure and affinities of the Hedyloidea: a new concept of the butterflies. Bulletin of the British Museum of Natural History 53: 251-286.

SCOBLE MJ (1995) The Lepidoptera form, function and diversity. Oxford University Press, Suffolk, England. 404 pp.

SCOBLE MJ (1999) Geometrid moths of the world: a catologue (Lepidoptera: Geometridae). Volumes 1 y
2. The Natural History Museum. CSIRO Publishing, Collingwood, Victoria, Australia. 1016 pp.

STEHR FW (1987) Immature insects. Kendall-Hunt Publishing Company, Dubuque, Iowa, USA. 754 pp.

WALKER F (1862) List of the specimens of Lepidoptera insect in collection of the British Museum 24: 1076.

WALKER F (1863) List of the specimens of Lepidoptera insect in collection of the British Museum 26:1644.

WARREN W (1894) New genera and species of Geometridae. Novitates Zoologicae 1: 366-466.

ZAKVATKIN V (1969) Embriology and systematics of leaf beetles Coleoptera, Chrysomelidae. Evolution Galerucinae-Chrysomelidae. Australian Journal of Science 32: 208-212.

\section{ANEXO 1}

Clave para separar las especies chilenas del género Syncirsodes según la maculación alar y armadura genital de los adultos

Key to Chilean species of the genus Syncirsodes based on maculation and genitalia

1. Alas con una escotadura en el área media del margen externo, antenas simples (hembras) 2

1' Alas sin escotadura, margen externo liso, antenas pectinadas (machos) .... 9

2.(1) Superficie dorsal de las alas amarillenta

2' Superficie dorsal de las alas de otro color

3.(2) Alas anteriores amarillo pálido, banda medial levemente más oscura, alas posteriores de color más pálido y homogéneo (Fig. 1R). Colliculum esclerosado en el centro con forma romboidal (Fig. 5A) S. straminea

3 Alas anteriores amarillentas, banda medial, anaranjada o castaño claro, una mancha anaranjada más intensa rodea la zona de celda discal, dos manchas circulares en la zona medial del borde externo; alas posteriores más pálidas (Fig. 1D). Colliculum en forma de cinturón, ancho 2 veces su largo (Fig. 2A) S. distictaria

4.(2') Superficie dorsal de las alas anaranjadas a castaño rojizo 5

4. Superficie dorsal de las alas de otro color

5. (4) Alas anaranjadas, abigarradas o no de escamas más oscuras, banda adtermi- nal gris pálido, dos manchas circulares bajo la zona de la escotadura, banda medial conspicua amarilla, banda antemedial más oscura. Alas posteriores más pálidas (Fig. 1H). Colliculum en forma de cinturón, ancho 2 veces su largo (Fig. 3A) S. hyadesi

5. Alas castañas rojizo, ductus bursae levemente esclerosado 6

6.(5') Alas castaño rojizo, banda medial conspicua levemente más oscura, alas posteriores similares a las anteriores (Fig. 1Ñ). Colliculum esclerosado en el centro de forma irregular (Fig. 4A) S. primata

6' Alas castaño rojizo, banda medial conspicua, banda postmedial, cruzada por una línea oblicua oscura a gris, alas posteriores similares a las anteriores (Fig. 1T). Colliculum esclerosado de forma romboidal (Fig. $5 \mathrm{~A})$ S. straminea

7.(4) Alas bicolor, área de intersección de la línea oblicua y la banda medial amarillo pálido (Fig. 1N). Colliculum esclerosado de forma irregular (Fig. 4A) S. primata

Alas de un solo color 8 Alas abigarradas con escamas oscuras 13 
8.(7') Alas de color rosado grisáceo, banda medial más oscura, línea adterminal más oscura, línea del área basal irregular y oscura (Fig. 1M). Colliculum esclerosado de forma irregular (Fig. 4A) S. primata

8

Alas de color gris oscuro o gris claro (similar a Fig. 1Q), banda medial más oscura, línea oblicua más oscura, línea del área basal irregular y oscura. Colliculum esclerosado de forma irregular (Fig. 4A) S. primata

9. (1') Superficie dorsal de las alas amarillentas 10

9' Superficie dorsal de las alas anaranjadas 12

10.(9) Línea oblicua castaña cruza el ala desde el primer tercio del margen costal hasta la zona media del margen interno (Fig. 1A). Cerdas en el área del cucullus, uncus triangular (Fig, 2B) S. distictaria

10. Cucullus con microespinas o cerdas 11

11. Uncus subtriangular; valvas subtriangulares, escasas cerdas en el sector del cucullus (Fig. 5B) S. straminea

11' Uncus espatulado; valvas subcuadrangulares, un grupo de espinas largas y cónicas en el margen costal de las valvas (Fig. 3B) S. hyadesi

12.(9') Como Fig. 1O. Gnathos en forma de "V" (Fig. 4B) S. primata

12' Como Fig. 1E-1G. Uncus espatulado (Fig. 3B) S. hyadesi

13.(7") Alas anteriores castaño oscuro, con dos manchas oscuras circulares en la zona media del margen externo, toda la superficie abigarradas con escamas oscuras, alas posteriores más claras (Fig. 1B). Cerdas en el cucullus, uncus triangular (Fig. 2B) S. distictaria

13' Alas castaño claro abigarradas con escamas castaño oscuro 14

14.(13') Banda medial muy notoria de color castaño oscuro, banda postmedial amarillenta, línea adterminal gris o amarillenta (Fig. 1P). Gnathos en forma de "V" (Fig. 4B) ............ S. primata

14 Banda medial insconspicua, color de las alas uniforme .............................. 15

15.(14') Uncus espatulado, valvas subcuadrangulares, un grupo de espinas largas y cónicas en el margen costal de las valvas (Fig. 3B) S. hyadesi

15 Alas castaño claro, abigarradas de escamas castaño oscuro (Fig. 1I). Gnathos en forma de "V" (Fig. 4B).... S. primata 